\title{
Drug encapsulated polymeric microspheres for intracranial tumor therapy: A review of the literature 2 , 败访
}

\author{
J. Alaina Floyd ${ }^{\mathrm{a}}$, Anna Galperin ${ }^{\mathrm{b}}$, Buddy D. Ratner ${ }^{\mathrm{a}, \mathrm{b}, *}$ \\ a University of WA, Department of Chemical Engineering, Seattle, USA \\ ${ }^{b}$ University of WA, Department of Bioengineering, Seattle, USA
}

\section{A R T I C L E I N F O}

Available online $\mathrm{xxxx}$

\section{Keywords:}

Drug delivery

Microspheres

Gliomas

Chemotherapeutics

Localized therapy

Emulsion

5-Fluorouracil

Paclitaxel

\begin{abstract}
A B S T R A C T
Despite intensive surgical excision, radiation therapy, and chemotherapy, the current life expectancy for patients diagnosed with glioblastoma multiforme is only 12 to 15 months. One of the approaches being explored to increase chemotherapeutic efficacy is to locally deliver chemotherapeutics encapsulated within degradable, polymeric microspheres. This review describes the techniques used to formulate drug encapsulated microspheres targeted for intracranial tumor therapy and how microsphere characteristics such as drug loading and encapsulation efficiency can be tuned based on formulation parameters. Further, the results of in vitro studies are discussed, detailing the varied drug release profiles obtained and validation of drug efficacy. Finally, in vivo results are summarized, highlighting the study design and the effectiveness of the drug encapsulated microspheres applied intracranially.
\end{abstract}

(c) 2015 Elsevier B.V. All rights reserved.

\section{Contents}

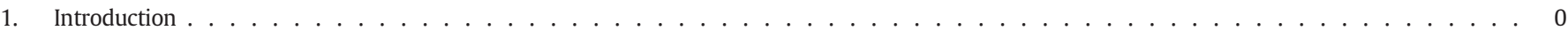

2. Microsphere formation methodology . . . . . . . . . . . . . . . . . . . . . . . . . . . . . . 0

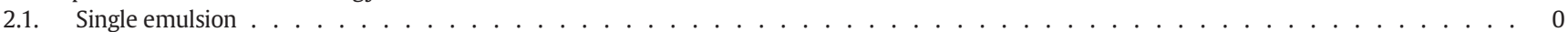

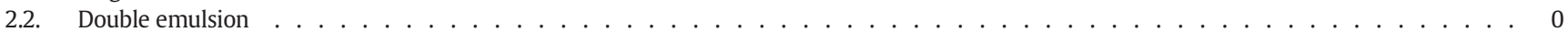

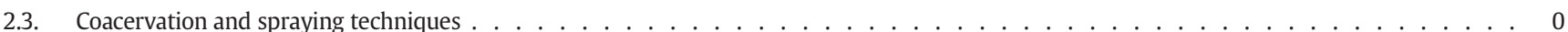

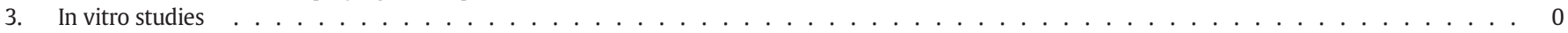

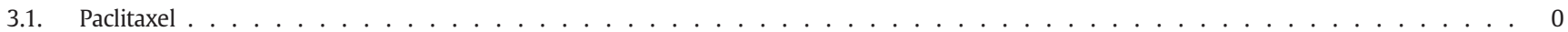

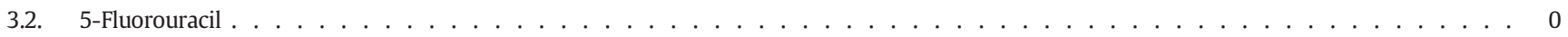

3.3. Doxorubicin, carboplatin, and $\mathrm{BCNU} \ldots \ldots \ldots \ldots$

3.4. Other small molecule chemotherapeutics . . . . . . . . . . . . . . . . . . . . . . . . . . . . . . 0

3.5. Endogenous inhibitors, bone morphogenetic protein-7, interleukin-2, and recombinant adenovirus . . . . . . . . . . . . . . . . 0

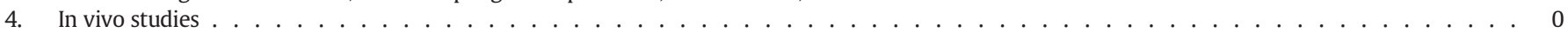

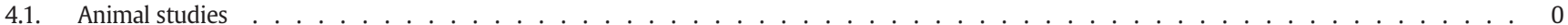

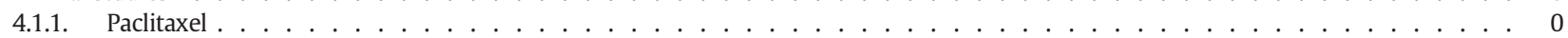

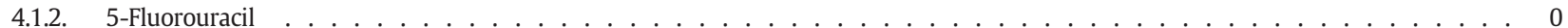

4.1.3. Carboplatin and BCNU . . . . . . . . . . . . . . . . . . . . . . . . . . . . . . . . . . . . . . . . . . . . . . 0

4.1.4. Other small molecule chemotherapeutics . . . . . . . . . . . . . . . . . . . . . . . . . . 0

4.1.5. Endogenous inhibitors and interleukin $-2 \ldots \ldots \ldots \ldots$

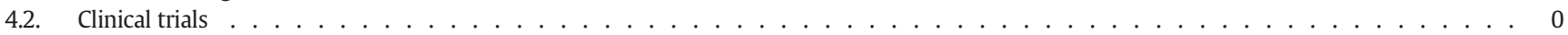

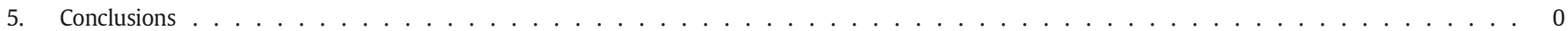

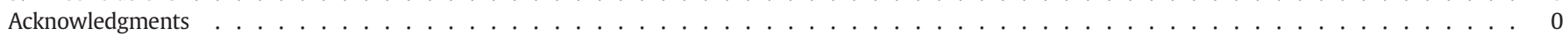

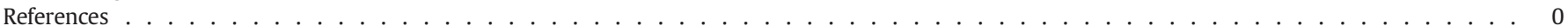

\footnotetext{
Color copies of figures are available in electronic form.

is th This review is part of the Advanced Drug Delivery Reviews theme issue on "Editor's Collection 2015".

* Corresponding author at: 3720 15th Ave NE, Box 355601, Seattle, WA 98195. Tel.: +1 206543 2250; fax: +1 2065433778.

E-mail address: ratner@uweb.engr.washington.edu (B.D. Ratner).
} 


\section{Introduction}

Malignant tumors of the brain and spinal cord are diagnosed in over 20,000 men and women in the United States in 2014 [1,2]. Over half of these will prove fatal to the patient. The fastest growing and most common malignant tumors originate in the glial or support cells of the brain. These tumors include astrocytomas, oligodendrogliomas, ependymomas, and the most common and aggressive type, glioblastoma multiforme [2,3]. Ultimately, despite treatments including a combination of surgical resection, chemotherapy, and radiation [4], these tumors will regrow. After diagnosis, a 12-15 month survival period is expected $[5,6]$. This low survival rate is attributed to such factors as tumor drug resistance, intracellular drug metabolism, and limited drug uptake, in part due to the major obstacle of the blood brain barrier which prevents many systemic therapies from reaching the brain [7-9]. In addition, complete tumor removal is difficult to achieve without causing brain damage. Tumor reccurrence is generally within $2 \mathrm{~cm}$ of the primary tumor site, in part due to tumor remnants, emphasizing the need for a localized and sustained delivery of therapeutic agents to treat the new growth and bypass the blood brain barrier [10].

To address the need for a localized drug delivery system to target tumor regrowth, the scientific community has developed different approaches for intracerebral therapy including implantable reservoirs, biodegradable drug carriers, and convection-enhanced delivery $[6,11$, 12]. Currently, the only FDA approved intracerebral therapy is the application of GLIADEL $®$ wafers at the site of tumor resection. These wafers are a degradable polymeric matrix composed of 1,3-bis(pcarboxyphenoxy)propane (CPP) and sebacic acid (SA), loaded with carmustine [13]. Locally implanted, GLIADEL $®$ wafers degrade over time, delivering the payload in a controlled manner to the tumor site. Combined with tumor resection and radiation therapy, the GLIADEL ${ }^{\circledR}$ wafers were shown to increase patient survival by approximately two months [14].

The use of biodegradable drug carriers like GLIADEL ${ }^{\circledR}$ wafers are a promising avenue for intracranial delivery due to their steady-state release, minimized systemic side effects associated with localized delivery, and increased patient convenience from the therapy due to drug release over several weeks to months. However, polymeric wafers are just one type of biodegradable drug carrier.

Polymeric microspheres and nanoparticles are alternative delivery vehicles for a localized, glioma therapy. While the systemic administration of polymeric, drug-loaded nanoparticles, ranging from 10 to $1000 \mathrm{~nm}$ in size, is minimally effective due to a low percentage of particles that successfully cross the blood brain barrier and enter the brain, the localized administration of "penetrating" nanoparticles have demonstrated therapeutic efficacy in vivo [15-17]. Microspheres are also applied after tumor resection, overcoming the blood brain barrier and allow for a slow, long term release of a chemotherapeutic. However, microspheres offer the potential for greater suspension stability, high drug loadings, and lower burst release due to their lower surface area to volume ratio [18]. Thus, there are positive aspects to both microsphere and nanoparticle delivery of therapeutics to brain tumors. However, this article will focus on microspheres for brain cancer drug delivery.

While microspheres for controlled drug delivery have been reviewed [19-22], their potential use for glioma treatment has not been considered in an overview article. Herein, the methodologies for forming degradable, drug loaded polymeric microspheres for the localized treatment of brain tumors will be reviewed together with in vitro and in vivo results, encompassing the various small and large molecule therapeutics that have the potential for positively impacting brain tumor therapy.

In this review article, we are not necessarily advocating microsphere delivery of cancer therapeutic drugs as the ideal treatment modality, but rather summarizing the published literature on this widely used strategy for treatment. The fact that there is such an extensive literature on microsphere drug delivery for treating brain tumors suggests that many leading practitioners and researchers consider microsphere deliver a viable contender for advanced brain tumor therapies.

\section{Microsphere formation methodology}

While there are many reviews detailing the formulation and characterization of microspheres for drug delivery purposes [19-21, $23,24]$, this section endeavors to provide an overview of the techniques used to prepare chemotherapeutic encapsulated microspheres specifically for intracranial tumor therapy. In addition, this section provides insight into the parameters that can be manipulated to achieve microspheres with tailored properties, e.g., size, morphology, drug loading, encapsulation efficiency, etc.

\subsection{Single emulsion}

The single emulsion solvent evaporation (or solvent extraction) technique, also called the oil-in-water $(\mathrm{O} / \mathrm{W})$ emulsion, is a common method for forming drug encapsulated polymeric microspheres for intracerebral therapies [25-43]. As shown in Fig. 1, the polymer to form the microsphere, poly(lactic-co-glycolic acid) (PLGA) or poly(lactic acid) (PLA) for example, is dissolved in an organic solvent, usually methylene chloride, along with the drug to be encapsulated. This "oil" solution is added to an aqueous solution containing a stabilizer, e.g., poly(vinyl alcohol) (PVA), and homogenized. The organic solvent is then removed by evaporation and the drug encapsulated microspheres are recovered. A variation of the single emulsion method can use an acetone/mineral oil emulsion instead of the organic and water system [30].

A variety of chemotherapeutics including temozolomide, bischloroethylnitrosourea (BCNU), paclitaxel, 5-fluoruracil (5-FU), and 5iodo-2'-deoxyuridine (IUdR) have been encapsulated in polymeric microspheres by the single emulsion technique for intracranial tumor therapy $[26-31,38]$. Based on the polymer to oil ratio, homogenizing speed, drug concentration, stabilizer concentration, and other formulation parameters, the sphere size, morphology, drug loading and encapsulation efficiency can be tailored.

For example, Zhang et al. has shown that both polymer concentration and stirring rate can affect the size of temozolomide loaded PLGA microparticles [27]. For a PLGA concentration increased from 5 to $13.33 \%(\mathrm{w} / \mathrm{v})$, microparticle size changed from 55.2 to $73.6 \mu \mathrm{m}$, respectively. This is attributed to the increase in viscosity that comes from

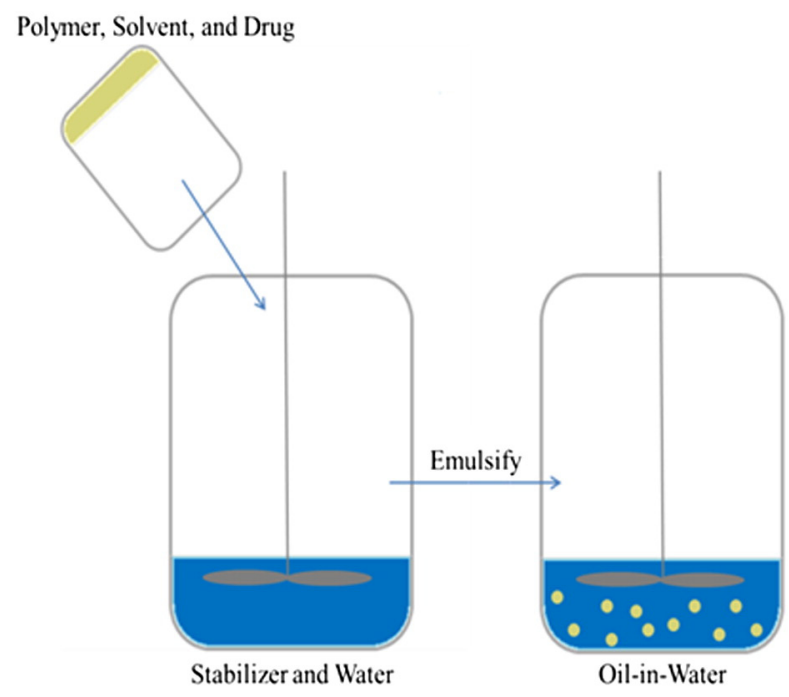

Fig. 1. Schematic of the oil-in-water $(\mathrm{O} / \mathrm{W})$ solvent evaporation technique for microsphere formation. 
increasing the polymer concentration; the more viscous solution shears differently during the emulsifying process, producing larger particles. In addition, the viscous environment increases the chance of particles fusing together during the formation process [27]. Manipulation of the emulsifying speed from 400 to $800 \mathrm{rpm}$ caused a decrease in particle size from 71.7 to $54.3 \mu \mathrm{m}$, respectively, due to the greater shear rates at the higher speed. Reza et al. showed the same trend for IUdR loaded PLGA microspheres, where an increase in stirring rate from 300 to $1000 \mathrm{rpm}$ decreased the mean particle size from 89.8 to $38.2 \mu \mathrm{m}$, respectively [38].

Chen et al. conducted studies on polymer concentration and its effects on carboplatin drug loading [30]. With an increase in PLGA concentration from 6 to $8 \%(\mathrm{w} / \mathrm{v})$, both the microsphere yield and drug loading increased. However, a critical point was reached where a further increase in concentration did not yield a higher drug loading. The solution became viscous enough to encapsulate the maximum amount of drug and a further increase in polymer concentration would cause a decrease in drug loading. Similarly, Gil-Alegre et al. has shown that there is a limit to the drug/polymer ratio for increasing encapsulation efficiency of BCNU loaded PLGA microspheres [29]. When the ratio was increased from 0.2 to 0.4 , the drug loading doubled and the encapsulation efficiency remained the same. However, for an increase to a 0.8 ratio, the drug loading did not change; instead, a decrease in encapsulation efficiency resulted. This indicates that there is a solubility limit that must be considered when encapsulating drugs with the $\mathrm{O} / \mathrm{W}$ solvent evaporation technique.

Changes in the initial drug loading have also been studied by Elkharraz et al. for paclitaxel loaded PLGA microspheres [26]. In this case, the initial drug loading effects were not as significant as those discussed above for BCNU. Paclitaxel is a highly lipophilic drug, which would minimize its diffusion to the external aqueous phase, resulting in an insignificant effect of drug loading changes on encapsulation efficiency. Rather, the noticeable effect was seen on particle size for changes in the stabilizer (PVA) concentration [26]. When the concentration of PVA increased from 1 to $5 \%(\mathrm{w} / \mathrm{v})$, the size of the microspheres decreased from $32-34 \mu \mathrm{m}$ to $17-19 \mu \mathrm{m}$, respectively. This was attributed to two effects of the increased PVA concentration: a decreased surface tension between the organic and aqueous phase and an increased external aqueous viscosity. Both would result in smaller microparticles being formed. The smaller microparticles resulting from the greater stabilizer concentration have a smaller drug diffusion pathway through the polymer, leading to a greater potential for loss to the outer, aqueous phase. This is demonstrated by the lower encapsulation efficiency for the higher stabilizer concentration formulations.

The effect of the physical size of the drug on drug loading and microsphere morphology was demonstrated by Boisdron-Celle et al. in 5-FU loaded PLGA microspheres [44]. Raw 5-FU drug crystals have a mean diameter of $247 \pm 95 \mu \mathrm{m}$. To be encapsulated in microspheres 20 $40 \mu \mathrm{m}$ in diameter, the crystals must be fragmented. While grinding the drug down to a fine powder enables encapsulation, there is a trade-off of a lower encapsulation efficiency because the smaller crystals readily diffuse out into the external aqueous phase during the microsphere formation, lowering the drug recovery. However, large crystals cannot be incorporated into the microsphere. In addition, the larger crystals led to misshapen microspheres with visible pores. This occurs because the larger crystals are initially on the surface but dissolve during the microsphere processing, leaving pores on the surface. The smaller drug crystals, in contrast, produced a smoother surface that is akin to the blank, drug-free microspheres.

A drawback to the oil-in-water emulsion is a low encapsulation efficiency for hydrophilic compounds that will preferentially diffuse out to the external, aqueous phase. For example, Reza et al. has shown that for IUdR loaded PLGA microspheres, an increase in the theoretical drug loading from 10 to $30 \%$ did not increase the actual loading (2$4 \%$ ) due to the hydrophilicity of IUdR [38]. This can be overcome by saturating the external aqueous phase with the drug in addition to having the drug in the internal, oil phase $[27,38]$. While only a $3 \%$ increase in drug loading was seen for IUdR, it was found for temozolomide loaded PLGA microspheres that a temozolomide-saturated PVA aqueous phase more than doubled the encapsulation efficiency [27].

\subsection{Double emulsion}

The double emulsion, solvent evaporation (or solvent extraction) technique also called the water-in-oil-in-water $(\mathrm{W} / \mathrm{O} / \mathrm{W})$ emulsion is commonly used to encapsulate hydrophilic compounds, overcoming the limitations of a single oil-in-water emulsion. As shown in Fig. 2 the drug to be encapsulated is dissolved in a small volume of water and added to an organic solution, frequently methylene chloride, containing the dissolved polymer. This is emulsified and creates the first water-in-oil emulsion $(\mathrm{W} / \mathrm{O})$. This $\mathrm{W} / \mathrm{O}$ emulsion is then added to an aqueous phase containing a stabilizer, normally PVA. This is also emulsified, forming the double emulsion of water-in-oil-in-water $(\mathrm{W} / \mathrm{O} / \mathrm{W})$. The organic layer acts as a barrier, preventing the hydrophilic drug from diffusing from the internal, aqueous phase to the external, aqueous phase [38]. The organic solvent then evaporates over time before the microspheres are recovered.

The double emulsion technique was used to create drug loaded polymeric microspheres for intracranial tumor therapy, encapsulating chemotherapeutics such as 5-FU, camptothecin, imatinib mesylate, Cterminal fragment of platelet factor 4 (PF-4/CTF), hemopexin (PEX), doxorubicin, radioiodinated IUdR, antisense oligonucleotides, and mitoxantrone [38,45-52]. Similar to the single emulsion technique, the double emulsion system offers flexibility with respect to its parameters such as polymer concentration, drug concentration, stabilizer type and concentration, homogenizing speed, etc. to tune the sphere size and morphology, drug loading, and encapsulation efficiency. For example, Reza et al. manipulated the oil phase composition to increase the drug loading of IUdR loaded PLGA microspheres for intracranial tumor therapy by adding both acetone and Span 40 to methylene chloride [38]. Acetone was used as a co-solvent, rapidly mixing in the aqueous phase and resulting in a faster formation of solid microspheres than with methylene chloride alone. Span 40 was added to the oil phase to improve the stability of the primary emulsion. These additives to the organic phase and the addition of bovine plasma to the inner water phase to bind the IUdR increased the actual drug content to an optimized $36.5 \%$ from initial experiments where values as low as $4 \%$

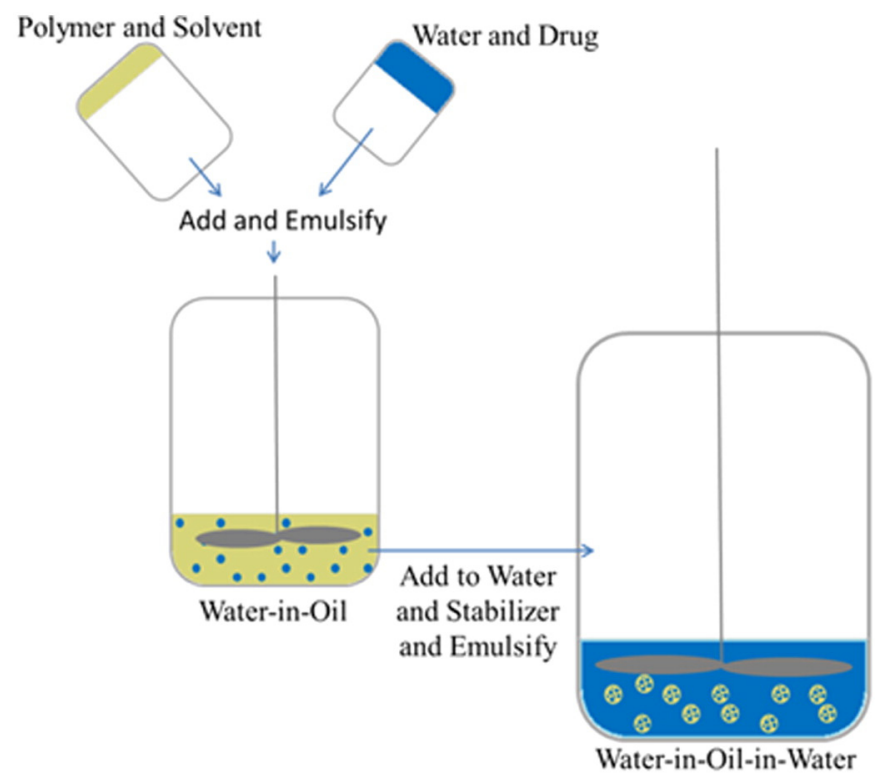

Fig. 2. Schematic of water-in-oil-in-water double emulsion, solvent evaporation technique for microsphere formation. 
were noted. The effect of stirring time was also studied for the IUdR loaded PLGA microspheres. Originally, the microspheres were stirred at $1000 \mathrm{rpm}$ for three hours, resulting in particles of approximately $35 \mu \mathrm{m}$ and a $4 \%$ drug loading. However, when the stirring time was shortened to $5 \mathrm{~min}$ at $1000 \mathrm{rpm}$ followed by a slow speed stirring at $500 \mathrm{rpm}$ for $1.5 \mathrm{~h}$ to evaporate the organic solvents, the drug loading and particle size increased to $24.2 \%$ and $39 \mu \mathrm{m}$, respectively [38].

The effect of stabilizer type on surface morphology of 5-FU loaded PLA and PLGA microspheres was demonstrated by Chandy et al. using either PVA or chitosan as the stabilizer [48]. While microspheres approximately $10-25 \mu \mathrm{m}$ in diameter were formed for both stabilizers, a difference in the surface morphology was detected. PVA led to the production of PLA microspheres with a micro-porous structure and open channels at the surface, while chitosan produced fewer pores leading to smoother and non-porous PLA and PLGA microspheres. This smoothening affect is attributed to the hydrophilic nature of chitosan.

The $\mathrm{W} / \mathrm{O} / \mathrm{W}$ emulsion has also been used to encapsulate molecules beyond the traditional small molecule chemotherapeutics for glioma treatment. These include oligonucleotides and endogenous inhibitors $[45,46,51]$. Hussain et al. has successfully encapsulated antisense oligonucleotides (AODNs) in PLGA microspheres to be co-administered with 5-FU [51]. AODNs inhibit gene expression at the mRNA level, downregulating the expression of disease-causing proteins. However, AODNs are limited by their short half-life in vivo. With the use of polymeric carriers, the lifetime can be extended by protecting the AODNs until degradation of the microspheres and subsequent release.

Endogenous inhibitors such as PF-4/CTF and PEX, which are both proteins, have been encapsulated in PLGA microspheres by Benny et al. $[45,46]$. An approximate drug loading of 0.63 and $0.13 \%$ was achieved for PF-4/CTF and PEX, respectively [45]. The PF-4/CTF encapsulation efficiency was modestly dependent on the PLGA type, whether the polymer had a free carboxyl (uncapped) or an esterified (capped) end group. The uncapped, more hydrophilic PLGA had an encapsulation efficiency of $52.8 \pm 7 \%$ while the hydrophobic, capped PLGA was $69.8 \pm$ $8 \%$ [46]. The successful encapsulation of these endogenous inhibitors points toward a new range of chemotherapeutics different from the traditional small molecules that might in the future be used for the localized treatment of brain cancer using polymeric microspheres.

\subsection{Coacervation and spraying techniques}

While coacervation and spraying techniques such as spray drying and electrohydrodynamic atomization (EHDA) are used to form polymeric microspheres for drug delivery, there are few reports that utilize these techniques for intracranial therapy application. These studies are reviewed here.

Coacervation or phase separation involves dissolving the polymer in an organic solvent that contains the drug to be encapsulated. This mixture is added to an organic nonsolvent such as vegetable oil to induce polymer phase separation. The polymer solvent is extracted during this period before another nonsolvent such as hexane or heptane is added to achieve final sphere hardening, followed by microspheres collection and washing $[21,23,41]$. Emerich et al. has used coacervation to encapsulate carboplatin in PLGA microsphere for intracranial tumor therapy [40-42,53], formulating carboplatin encapsulated PLGA microspheres at a $10 \%$ drug loading [41].

A typical spraying technique is spray drying, where the polymer and drug are dissolved in a solvent and passed through a spray dryer. A stream of heated air breaks the polymer solution into droplets, forming microspheres that are dried and collected $[21,54,55]$. Spray drying has been successfully used to encapsulate paclitaxel and doxorubicin (DOX) in PLA and PLGA polymeric microspheres for glioma treatment $[54,55]$. Lin et al. formulated DOX encapsulated PLGA microparticles with sizes of $2.18 \pm 0.64$ to $3.74 \pm 0.17 \mu \mathrm{m}$ depending on the formation parameters, such as the use of the additive Pluronic and different copolymer ratios of PLGA. The described monodisperse size distribution highlights the controlled particle size that is characteristic of spray drying $[21,22,55]$. It was shown that increasing the ratio of lactide to glycolic acid in the PLGA composition from $65 / 35$ to $85 / 15$ increased the encapsulation efficiency from $59.6 \%$ to $73.8 \%$, respectively. This is attributed to a better interaction of the organic solvent with the polymer containing a higher lactide fraction, the more hydrophobic copolymer.

EHDA or electrospraying also has the benefit of an efficient control for microsphere size, creating a monodisperse distribution. In EHDA, the polymer and drug are dissolved in a solvent before passing through a nozzle and a ring that has a large potential difference applied using high voltage generators. The solution is pumped through the nozzle, forming a liquid cone with a thin jet stream extending from its apex. This jet stream is broken up into monodisperse droplets. A stream of nitrogen can be used for solvent evaporation or a corona discharge system for particle neutralization during this time [56-58].

EHDA was applied to encapsulate paclitaxel in PLGA, PLA, and poly(E-caprolactone) (PCL) and cisplatin in PLA and PLA/PLGA composite microspheres for a localized glioma treatment [56-58]. Xie et al. tuned various parameters such as polymer concentration, organic solvent, and flow rate to achieve paclitaxel encapsulated PCL and PLGA microspheres [56]. For example, increasing PCL concentration increased particle size and smoothed surface morphology, resulting in particles of defined spherical shape (Fig. 3). Particle size was also tuned by the polymer solution flow rate, with the size decreasing from $11.1 \pm 0.8$ to $4.9 \pm 0.8 \mu \mathrm{m}$ by decreasing the flow rate from 3 to $0.5 \mathrm{~mL} / \mathrm{h}$, respectively. Overall, encapsulation efficiencies of approximately 7883\% in PCL and PLGA microspheres were achieved using EHDA [56].

\section{In vitro studies}

This section reviews the in vitro studies that have been conducted to determine the drug release profile and efficacy of polymeric microspheres encapsulating a variety of chemotherapeutics targeted for intracerebral glioma therapy.

\subsection{Paclitaxel}

Paclitaxel is a hydrophobic chemotherapeutic originating from the bark of the pacific yew tree, Taxus brevifolia [59]. This drug is a mitotic inhibitor, promoting the formation and stabilization of microtubules, which inhibits mitosis and leads to cell apoptosis [54]. While paclitaxel has been used to treat other cancer types such as ovarian and breast cancer, systemic delivery of this drug for glioma treatment has failed because of the low levels of paclitaxel that reach the gliomas due to poor penetration of the blood brain barrier $[59,60]$. Therefore, paclitaxel encapsulated microspheres are being studied for localized therapeutic agent delivery to brain tumors.

The release profile of paclitaxel from microspheres was tailored by controlling many parameters such as polymer type, fabrication technique, drug loading content, stabilizer concentration, and the use of additives to control porosity. For example, Naraharisetti et al. utilized two different formation techniques, spray drying and EHDA, to create two distinct release profiles of paclitaxel using PLGA as the microsphere carrier [59]. The spray drying technique resulted in microspheres that had a sustained, first-order release over 60 days while the EHDA technique had a pseudo-zero order release for 28 days after an initial burst for the first two days. In addition, PEG was added to the spray dried microspheres as a porosity modifying additive. This addition resulted in a faster and fuller release than with PLGA microspheres alone [59]. Similarly, Li et al. also demonstrated the effect of using PEG as an additive on paclitaxel release from polilactofate microspheres [60]. In this study, paclitaxel encapsulated polilactofate microspheres were pressed into disks with or without PEG. While the free microspheres resulted in the fastest drug release in vitro, the microspheres pressed into disks had a significantly slower release profile (Fig. 4). 

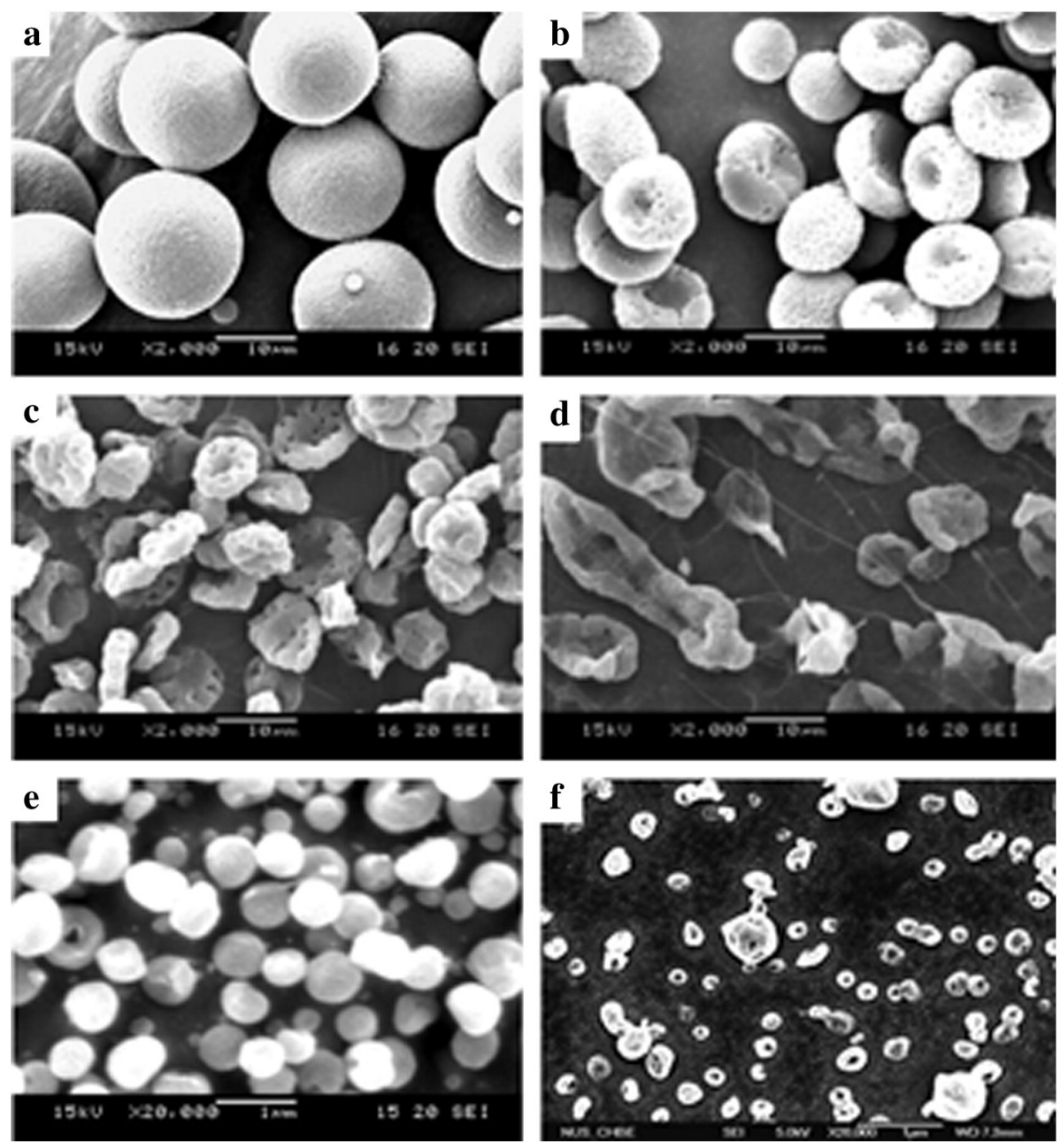

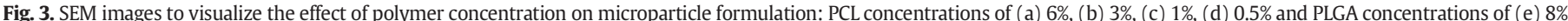

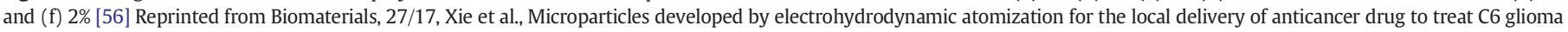
in vitro, 3321-3332, 2006, with permission from Elsevier.

The addition of PEG resulted in a concentration dependent rapid liquefaction of the disk; higher concentrations of PEG released the microspheres sooner for a faster drug release than disks without PEG.

The reverse effect, slowing the drug release rate, was achieved by Ranganath et al. by entrapping paclitaxel encapsulated PLGA microspheres in an alginate bead [58]. The alginate bead with free paclitaxel had a $100 \%$ release within two days; however, a 60 day sustained release with minimal initial burst was achieved by using drug encapsulated PLGA microspheres entrapped in the alginate bead. Additionally, the initial burst release was further retarded by having a denser packing of microspheres in the alginate bead, reducing the porosity and inhibiting diffusion of the solvent into the bead.

The effect of stabilizer concentration on the drug release profile was studied by Elkharraz et al. for paclitaxel encapsulated PLGA microspheres [26]. An increase in PVA concentration resulted in a decrease in sphere diameter due to the decreased surface tension between the organic and aqueous phase and an increased external aqueous viscosity. Subsequently, the sphere size had an effect on the drug release, with the smaller spheres having a faster release rate due to the decreased diffusion pathway lengths, regardless of the drug loading studied (Fig. 5).

Drug loading is an additional factor that affects the release rate of paclitaxel when all other parameters are held constant. Song et al. demonstrated that $5 \%$ paclitaxel loaded PLGA microspheres released paclitaxel slower than a $10 \%$ paclitaxel loaded microspheres [54]. At about day 60 , approximately $80 \%$ accumulated release was achieved for the $10 \%$ drug loading versus an approximate $40 \%$ release for the lower loading. This difference in the release rate was attributed to the drug concentration gradient from inside to outside that drives the diffusion and subsequent release from the microspheres. A similar effect of drug loading on the release rate was likewise observed by Xie et al. [56]. Paclitaxel loaded PLGA microspheres with a drug loading of 7.9 and $15.8 \%$ were studied and a slower release for the lower drug loading was observed over the 30 day experiment. The release of paclitaxel from PCL microspheres was also studied. PCL based particles released the drug significantly slower in comparison to PLGA formulations, approximately $30 \%$ and $55-60 \%$ at day 30 , respectively, most likely due to the higher hydrophobicity of PCL [56].

The bioactivity of paclitaxel released from PCL and PLGA microspheres was studied by using cell viability assays against rat $\mathrm{C} 6$ glioma cell lines and U251 human glioma cell lines [54,56,58]. The trend in all studies was increased cytotoxicity toward tumor cells with time, based on the sustained release of the drug from its polymeric carrier.

\subsection{5-Fluorouracil}

5-Fluorouracil (5-FU) is a hydrophilic, anti-metabolic drug. Due to its hydrophilic nature, it does not readily cross the blood brain barrier, making systemic delivery difficult. 5-FU is a drug of interest for targeting gliomas because it is a halogenated pyrimidine, making it a powerful radiosensitizer [31,34]. Therefore the stereotactic injection of polymeric microspheres loaded with 5-FU can be used as a way to overcome the 


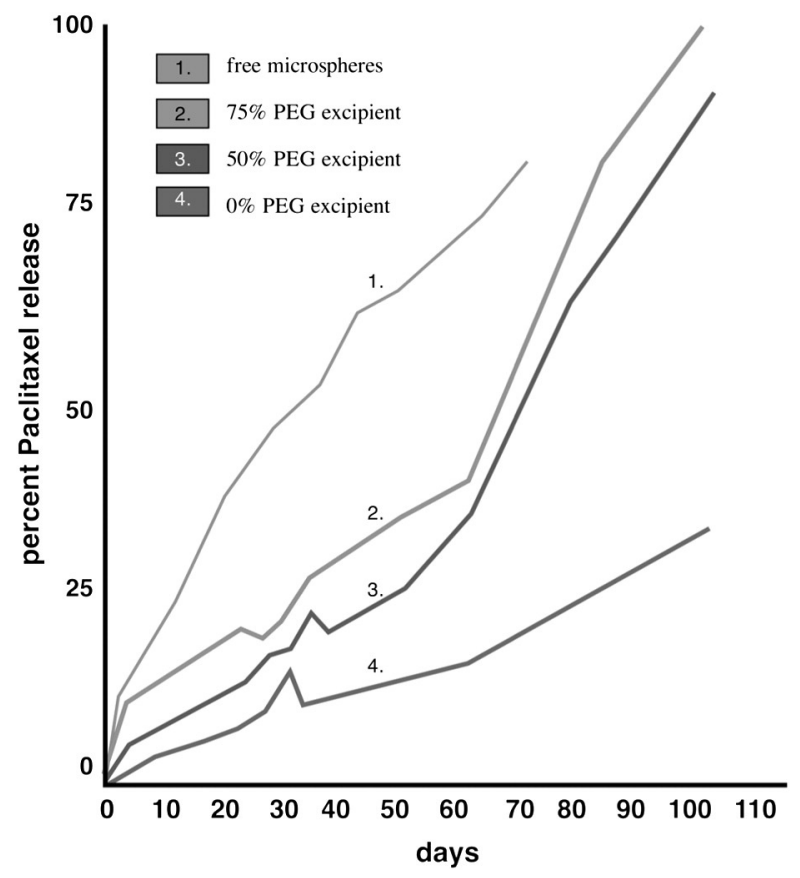

Fig. 4. Release of paclitaxel from free polilactofate microspheres (1) and from compressed polilactofate microspheres with increasing amounts of PEG-1000: 0\% (4), 50\% (3), and 75\% (2) PEG-1000. The figure was paraphrased from reference [60] due to concerns by the American Association of Cancer Research in reproducing the original figure.

blood brain barrier and provide a localized, sustained release to increase the effectiveness of radiation therapy.

The in vitro release profile of 5-FU was studied by a number of groups. They explored various formulation parameters such as polymer molecular weight, type and concentration, stabilizer type, and emulsion conditions to produce tailored release profiles $[34,36,43,44,48]$. As demonstrated by Menei et al. and Roullin et al., a typical release profile for 5-FU from PLGA microspheres is biphasic; there is an initial burst

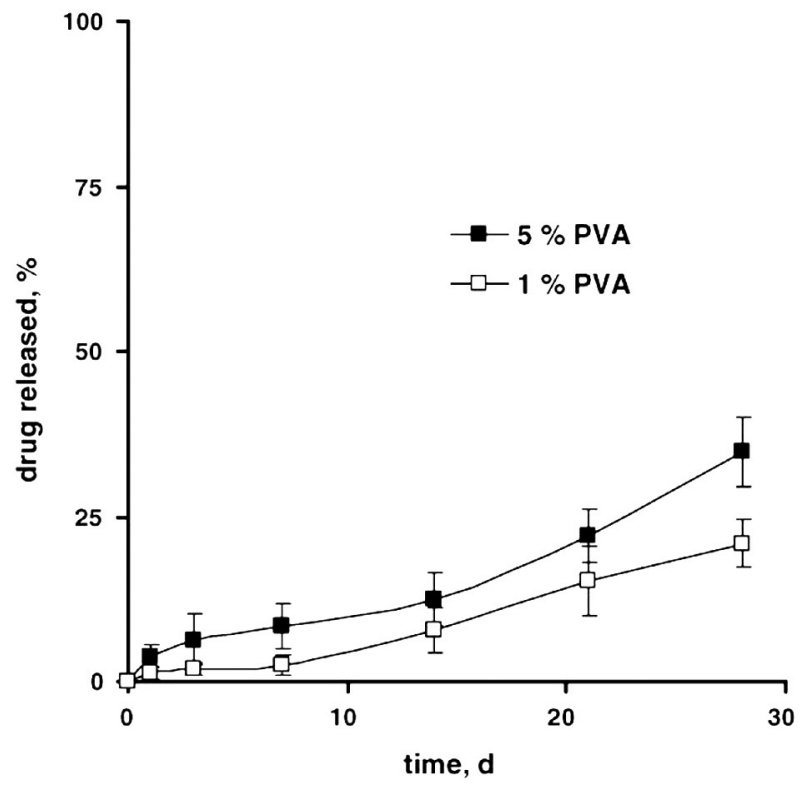

Fig. 5. The release of paclitaxel from PLGA microparticles in PBS formulated with different PVA concentrations [26] Reprinted from International Journal of Pharmaceutics, 314/2, Elkharraz et al., Paclitaxel-loaded microparticles and implants for the treatment of brain cancer: Preparation and physicochemical characterization, 127-136, 2006, with permission from Elsevier. phase around the first day followed by a sustained release over 20 days (Fig. 6) [34,36].

The same biphasic release behavior characteristic of the 5-FU loaded PLGA microspheres was reported by Chandy et al. for 5-FU loaded PLA microspheres [48]. These microspheres were formulated with either PVA or chitosan as the stabilizer. Regardless of stabilizer type, an initial burst release followed by a 30 day release was shown. It is important to note, however, that the stabilizer type did affect the rate of release: the chitosan stabilizer resulted in a slower release when compared to PVA, $72 \%$ and $82 \%$ release at day 30 , respectively. This is attributed to the different morphology of the two types of drug loaded microspheres: smooth and nonporous for chitosan and microporous for PVA. The porosity would enable the faster diffusion of the media into the sphere and subsequent faster release.

The size of the 5-FU drug crystals also plays a role in determining the drug release profile, as demonstrated by Boisdron-Celle et al. [44]. Two crystal sizes of 5 -FU, $11 \pm 9$ and $6.5 \pm 4.4 \mu \mathrm{m}$, were prepared and encapsulated within PLGA microspheres. The smaller crystal size had a markedly higher initial burst release, approximately $60 \%$ versus $50 \%$ release at $24 \mathrm{~h}$. In addition, the role of the polymer concentration was explored in this study, with a higher polymer concentration $(10.8 \%$ versus $5.8 \% \mathrm{w} / \mathrm{v}$ ) leading to a significantly slower release (18 days versus $48 \mathrm{~h}$ ). This is attributed to a decrease in microsphere porosity with increasing polymer concentration, which would limit diffusion. The study also explored the role of the organic to aqueous phase ratio on the release profile. An increase in the ratio from 1:61 to 1:33 resulted in a slower, more sustained release with a smaller initial burst effect. This was attributed to the distribution of 5-FU crystals inside the sphere. 5-FU loaded PLGA microparticles prepared at the 1:61 organic to aqueous phase ratio were uniformly loaded with the drug, leading to a faster release. The 1:33 ratio produced microspheres that did not have a uniform distribution; some of the 5-FU crystals were on the periphery and some were located in the core of the sphere, resulting in an initial burst followed by the sustained release through the polymer [44].

Hussain et al. studied the idea of co-delivery, either in separate microspheres or together in one carrier using 5-FU and antisense oligonucleotides (AODNs) [51]. AODNs inhibit gene expression by down regulating the expression of disease-causing proteins at the mRNA level. Specifically, the use of ADONs for this particular treatment of brain cancer is targeting the over-expressed epidermal growth factor receptor. When the drugs were individually entrapped in separate PLGA microspheres, 5-FU released faster in comparison to AODNs, with $\sim 80$ and $\sim 40 \%$ of each drug released after 35 days, respectively. However, when the drugs were co-entrapped, only $35 \%$ of the 5 -FU and $30 \%$ of the AODNs were released after 35 days. This slowing of release rate was attributed to hydrogen bonding between 5-FU and the AODNs, as suggested from results with an electrophoretic mobility shift assay.

Poly(methylidene malonate 2.1.2) (PMM 2.1.2) was used by Fournier et al. for 5-FU encapsulation. [43]. Compared to the widely used PLGA, PMM 2.1.2 is characterized by a longer degradation time and potentially slower release time. Three parameters that affect the release rate and drug loading of 5-FU were chosen in this work and studied with a $2^{3}$ full factorial design. These parameters included polymer molecular weight, polymer concentration, and emulsion time. The optimal release profile obtained was a $65 \%$ initial burst at $24 \mathrm{~h}$ followed by ongoing release 43 days later. The following parameter specifications were used: a higher polymer molecular weight $(24,135 \mathrm{~g} / \mathrm{mol})$ and a high polymer concentration with a low emulsion time (10\% w/v and $3 \mathrm{~min})$. The optimized, prolonged release was attributed to several factors. First, the higher polymer concentration and molecular weight resulted in a more viscous organic phase, preventing the diffusion of the drug crystals to the surface of the particles. Secondly, the shorter emulsion time afforded less time for the 5-FU crystals to escape to the external phase, resulting in a higher drug loading and again, fewer crystals localized along the periphery of 


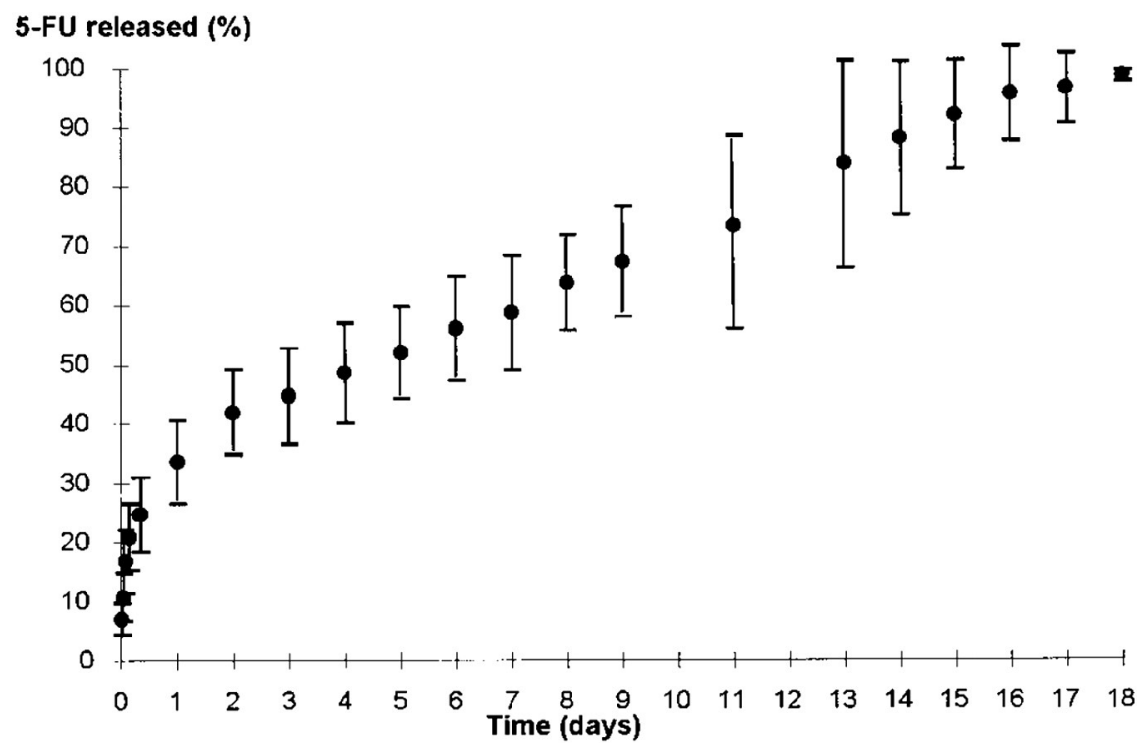

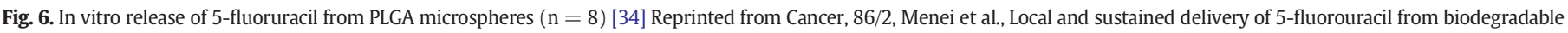
microspheres for the radiosensitization of glioblastoma-A pilot study, 325-330, 1999, with permission from John Wiley and Sons.

the sphere. Without this optimization, an approximate $90 \%$ initial burst release was detected within the first $24 \mathrm{~h}$.

\subsection{Doxorubicin, carboplatin, and $B C N U$}

Additional small molecule chemotherapeutics that have been encapsulated in polymeric microspheres for a localized glioma treatment include carboplatin, carmustine or bis-chloroethylnitrosourea (BCNU), and DOX $[29,30,41,50,55]$. Although these drugs are commonly used for other cancer types, their potential for glioma treatment has also been studied.

DOX is an anthracycline drug that interferes with the growth of the rapidly growing cancer cells. It inhibits further DNA and RNA biosynthesis, eventually causing cell death [55]. DOX is also a potent vesicant, causing tissue blistering. Therefore, a localized release is necessary to minimize its toxic side effects.

The choice of polymeric carrier had a significant effect on the release of DOX in vitro as demonstrated by Lin et al. using PLGA and PLGA-PLA composite microspheres [55]. For example, DOX loaded PLGA microspheres with differing copolymer ratios of the lactide and glycolide were studied. The more hydrophobic PLGA composition with an 85/15 lactide/glycolide ratio demonstrated a slower drug release and lower initial burst (60\%) compared to the more hydrophilic PLGA composition with a 65/35 lactide/glycolide ratio and an initial burst release of $70 \%$. It should also be noted that the particle size also attributed to the greater initial burst release and faster release for the more hydrophilic PLGA (65/35) spheres due to its smaller sphere size, $2.91 \pm 0.53 \mu \mathrm{m}$ versus $3.74 \pm 0.17 \mu \mathrm{m}$ (for PLGA 85/15). The most noticeable effect on burst release was detected comparing DOX loaded PLGA and PLGA-PLA composite microspheres, with a decrease from $60-75 \%$ to $36-48 \%$, respectively. It was suggested that the protective layer of PLA prevents the rapid release of the drug from the polymeric carrier, resulting in a more promising release profile. The DOX loaded PLGA microspheres were tested against $\mathrm{C} 6$ glioma cells and demonstrated a cytotoxic effect, indicating that DOX preserves its bioactivity through the encapsulation process [55]. In addition, DOX encapsulated microspheres slowed C6 glioma cell proliferation rate significantly when compared to the free drug after $96 \mathrm{~h}$, demonstrating the effectiveness of a sustained release.

The importance of the choice of carrier for drug release was also demonstrated by Arai et al. with DOX entrapped in a thermoreversible gelation polymer [(TGP, copolymer of poly ( $\mathrm{N}$-isopropylacrylamideco-n-butyl methacrylate) and poly(ethylene glycol) (PEG)] or encapsulated in PLGA microspheres that are entrapped in the TGP [50]. The free DOX entrapped in the TGP released $100 \%$ in 12 days while DOX loaded PLGA microspheres entrapped in TGP released $94 \%$ after 30 days. The effectiveness of this drug delivery system was tested on three cell lines: U87MG, LN229, and G55, as shown in Fig. 7. Overall, DOX loaded PLGA microspheres entrapped in TGP reduced tumor cell viability and maintained it at a low level for 30 days, unlike DOX in TGP, demonstrating the advantage of a sustained drug release.

Carboplatin is another potent, anticancer agent. Like DOX, it also has severe side effects such as leukopenia, peripheral neurotoxicity, and anemia when administered in high doses. This dose response makes localized delivery a necessity for use against brain cancer [30]. BCNU, another anticancer agent, is already used in polymeric wafers against brain tumors as an adjunct to radiation therapy after tumor resection [29]. While BCNU is lipid soluble and can readily pass through the blood brain barrier, it is rapidly metabolized, limiting its effectiveness. Therefore, a delivery system that can protect BCNU from being metabolized before reaching the tumor site is necessary.

As demonstrated by Chen et al., a release profile of carboplatin from PLGA microspheres exhibits two phases: diffusion during the first 10 days and then diffusion and bulk degradation, resulting in a faster release for days 12-22 [30]. The release media was varied by using either PBS or a $10 \%$ rat brain homogenate solution. While the release profiles are approximately the same during the first 10 days, controlled by diffusion, they differ after this time point. The rat brain homogenate resulted in a slower release rate, postulated by the authors to be due to the presence of proteins that adsorb on the surface and restrict water penetration into the spheres. A reduction greater than $30 \%$ was detected for the total release in the brain homogenate solution versus the PBS buffer.

Emerich et al. also studied the release of carboplatin from PLGA microspheres for a localized glioma delivery [41]. Carboplatin loaded PLGA microspheres demonstrated an initial burst of $13 \%$ in the first $24 \mathrm{~h}$ that was followed by a $100 \%$ release between 14 and 21 days. As a drug comparison, BCNU was also encapsulated in PLGA microspheres. Like carboplatin, this drug exhibited an initial burst of $10 \%$ before a full release was achieved in 14 days, more rapidly than the carboplatin. The difference in release rates can be attributed to the two different methods used to encapsulate the drugs (solvent evaporation for BCNU and coacervation for carboplatin) and the higher drug loading for BCNU (12.7\% versus $10 \%)$.

The efficacy of BCNU entrapped in PLGA microspheres with a 21 day release rate was tested by Gil-Alegre et al. against a U-373 MG human 

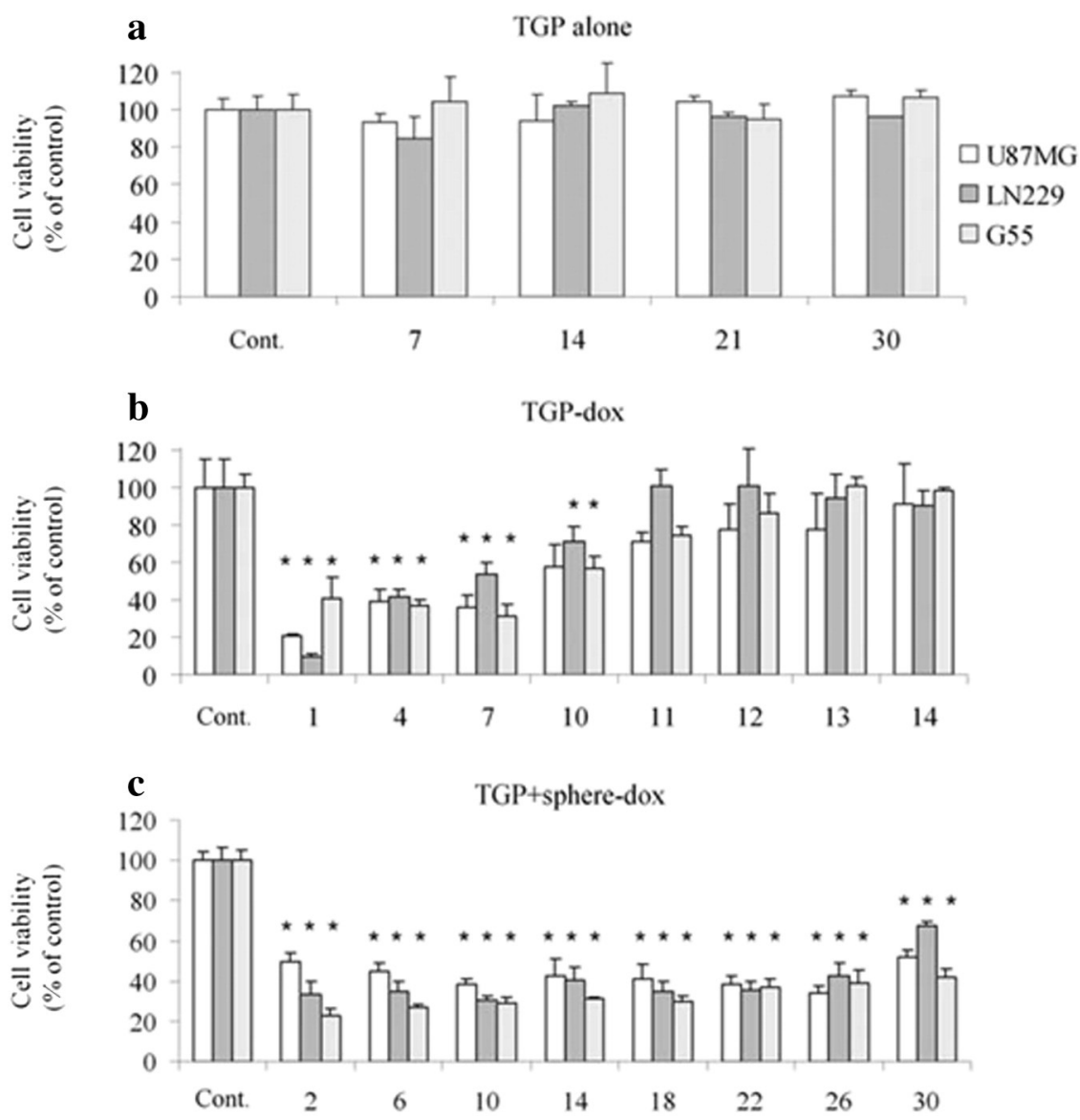

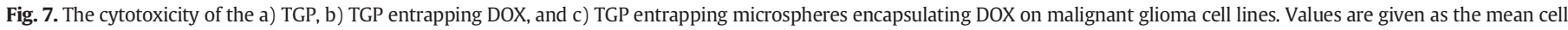

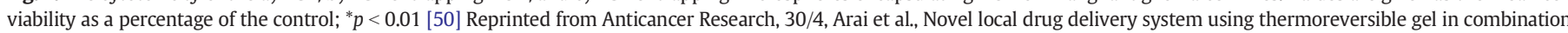
with polymeric microspheres or liposomes, 1057-1064, 2010, with permission from International Institute of Anticancer Research.

glioma cell line [29]. Toxic effects were produced during the 21 days of the study (approximately 90\% kill during the first two weeks with a decrease during the third week).

\subsection{Other small molecule chemotherapeutics}

Other common chemotherapeutics that are being applied to brain cancer are camptothecin, temozolomide, mitoxantrone, imatinib mesylate, cisplatin, gefitinib, radioiodinated IUdR, and phytocannabinoids $[27,38,47,49,52,57,61,62]$. The literature describing the delivery of each of these for glioma therapy will be presented here.

Camptothecin is an alkaloid that inhibits the progression of the Sphase of the cell cycle [49]. Camptothecin encapsulated PLGA microspheres were successfully formulated for a localized treatment of brain tumors. As demonstrated by Ozeki et al., the in vitro release profile lasted 30 days with an approximate $80 \%$ release $[49,63]$. Dispersing the drug encapsulated microspheres in a TGP produced a slightly prolonged but similar release (Fig. 8), indicating the release from PLGA was the rate limiting step rather than an effect of the TGP.

Temozolomide is an antineoplastic agent that is able to cross the blood brain barrier. However, its short half-life $(1.8 \mathrm{~h})$ necessitates the use of a delivery vehicle that protects the drug until release. This was achieved by encapsulating the drug within PLGA microspheres, as reported by Zhang et al. [27,64]. A 35 day release was demonstrated with the rate and initial release dependent on the drug loading [27]. The highest drug loading of $20 \%$ released faster and had the highest initial burst release compared to the lowest drug loading of $5 \%$ (Fig. 9). These temozolomide encapsulated PLGA microspheres were tested against C6 glioma cell lines, showing a sustained cytotoxicity for three days that was not apparent in the free drug form that was only effective for the first $12 \mathrm{~h}$.

In a different approach, Zhang et al. first absorbed the temozolomide on nanoparticulate hydroxyapatite and then encapsulated this combination in PLGA microspheres with the goal of slowing the release rate in vitro [64]. A three stage release (burst, diffusion, degradation) was seen for both temozolomide loaded spheres and temozolomide absorbed to hydroxyapatite encapsulated in PLGA spheres. However, the latter system demonstrated significantly slower release compared

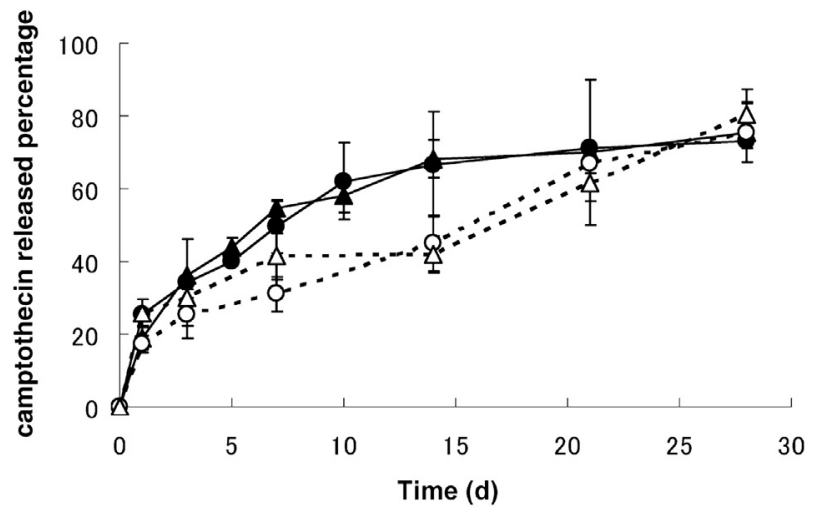

Fig. 8. Camptothecin release from PLGA microspheres in PBS (closed symbols, bold lines) and from PLGA microspheres dispersed in a TGP (open symbols, dotted lines) at camptothecin/PLGA ratios of $1 / 33$ (circles) and $1 / 50$ (triangles), $(n=5)$ [49]. Reproduced with permission from Chem. Pharm. Bull. Vol. 58 No. 9. Copyright 2010 The Pharmaceutical Society of Japan 


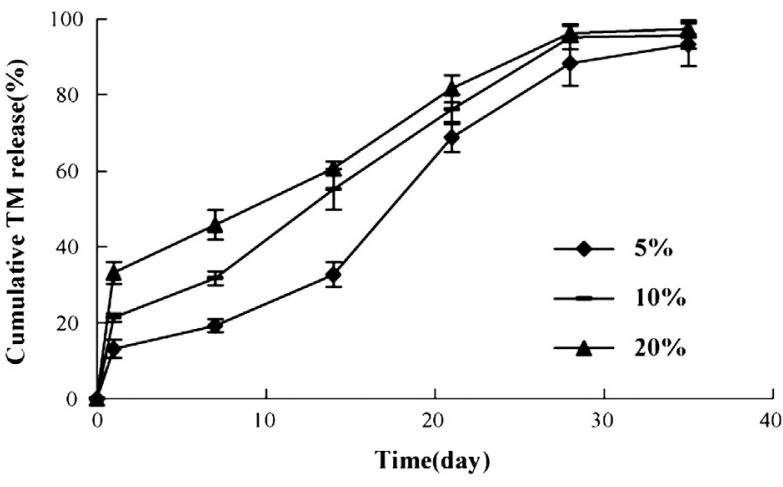

Fig. 9. The release profile of temozolomide from PLGA microparticles at 5, 10, and $20 \%$ drug loading ratio [27] Reprinted from International Journal of Pharmaceutics, 1-2, Zhang et al., Temozolomide/PLGA microparticles and antitumor activity against Glioma C6 cancer cells in vitro, 122-128, 2007, with permission from Elsevier.

to the formulation without the hydroxyapatite; the initial burst was reduced from $40 \%$ to $28-31 \%$ and the cumulative release was slower from day 2 to 14 . The reduction in release rate is attributed to the time it takes for the hydroxyapatite to dissolve and release temozolomide. When tested against a U87 glioma cell line, the temozolomide-hydroxyapatite-PLGA system reduced cell viability and increased cell apoptosis more efficiently over three and two days, respectively, than the temozolomide-PLGA spheres and free drug.

Another antineoplastic drug, mitoxantrone, is a synthetic anthracenedione that breaks the DNA strand and inhibits DNA topoisomerase II [52]. Yemisci et al. has encapsulated mitoxantrone in PLGA microspheres and detected a release of $55 \%$ at day 21 , followed by a near linear release through day 70 . These two phases in the release profile are attributed to the two mechanisms of release from PLGA microspheres: diffusion of the drug and then degradation and solubilization of the PLGA matrix [52].

Imatinib mesylate is a tyrosine kinase inhibitor that targets the activated Abl oncoprotein and part of the subgroup III receptor tyrosine kinase family [47]. While it is a standard of care for treating chronic myelogneous leukemia, the large amounts necessary to reach therapeutic levels in the brain justifies the need for a localized delivery. To fulfill this need, Benny et al. studied imatinib mesylate encapsulated in PLGA [47]. It was demonstrated that the release rate of imatinib mesylate from PLGA spheres was dependent on the ratio of the lactide to glycolide groups. The higher lactide content of 85/15 lactide/glycolide resulted in a slower release rate when compared to a 50/50 copolymer ratio. Release rate was tailored from 17 to 35 days depending on the copolymer ratio and was guided by the hydrophilicity/hydrophobicity of the PLGA composition. Drug bioactivity was tested using GL261 cell line, where the PLGA copolymer ratio of 50/50 had a drug release that inhibited cell viability by $57-65 \%$ for 12 days and the PLGA ratio of $85 / 15$ inhibited cell viability by $73-77 \%$ for 20 days.

Cisplatin is another chemotherapeutic that displays strong antitumor activity but has severe side effects. This drug binds to genomic DNA, eventually triggering the cytotoxic process leading to cell death [57]. Using both PLA and composite PLA-PLGA materials, Xie et al. successfully encapsulated cisplatin within microspheres and studied the released in vitro [57]. A significant initial burst release was detected for both types of polymeric carriers: $40 \%$ and up to $70 \%$ for PLA-PLGA composite and PLA microspheres, respectively. A slower release of the drug from the PLA-PLGA microspheres was detected in comparison to the PLA microspheres and is attributed to the core-shell structure of the composite spheres. Drug loading also affected the kinetics of cisplatin release from PLA microspheres with the $4 \%$ drug loading releasing more slowly compared to the $2 \%$ drug loading, possibly an effect of differences in particle morphology (Fig. 10). Using C6 glioma cells, the

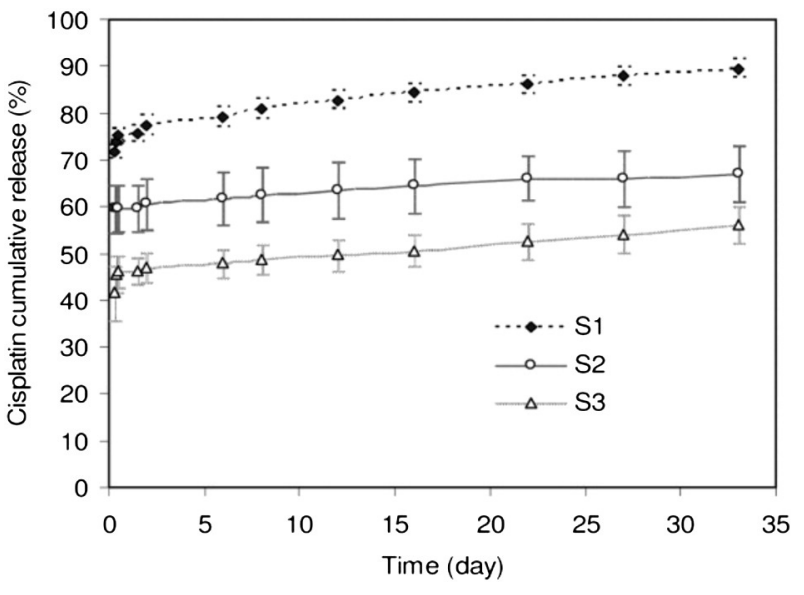

Fig. 10. Effect of drug loading on the in vitro release of cisplatin from PLA microparticles (S1: $2 \%$ and S2: $4 \%$ drug loading) and PLA/PLGA (30/70) microparticles (S3: $4 \%$ drug loading) [57] Reprinted from Journal of Biomedical Materials Research Part A, 85A/4, Xie et al., Biodegradable microparticles and fiber fabrics for sustained delivery of cisplatin to treat C6 glioma in vitro, 897-908, 2007, with permission from John Wiley and Sons.

cisplatin in both sphere types was proven to be cytotoxic with increasing incubation time and increasing concentration.

Gefitinib is an epidermal growth factor receptor tyrosine kinase inhibitor that prevents uncontrolled cell proliferation approved for use against non-small cell lung cancers [65]. Floyd et al. has encapsulated this chemotherapeutic in PLGA microspheres to achieve a 40 day release in vitro [61]. The release was moderate through day 20 followed by an increase in the release rate before completing at day 40 , attributed to diffusion and polymer bulk degradation, respectively. To note, the authors have proposed a system with multiple types of drug loaded microspheres formulated in an aqueous, degradable poly $(\mathrm{N}-$ isopropylacrylamide) (PNIPAM) solution for application via aerosolization to the tumor resection cavity. When tested in vitro, the microspheres in the PNIPAM matrix delayed gefitinib release by 4 6 days in comparison to microsphere release. We propose that the PNIPAM solution spray that adheres in a conformal manner to the irregular brain surface after surgical excision of the primary tumor mass will reduce the diffusional barrier to drug penetration since the suspended microspheres are held in intimate contact with the brain tissue [66].

Radioiodinated IUdR ( ${ }^{125}$ IUdR) causes cell disruption when taken into the cell nucleus over the short range of the low energy Auger

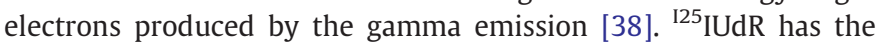
potential to be a therapy that can selectively kill brain tumor cells. Reza et al. encapsulated ${ }^{125}$ IUdR in PLGA microspheres and demonstrated an initial burst of 30-40\% followed by a diffusion sustained release for 35 days in vitro [38].

Cannabinol (CBD) and $\Delta^{9}$-Tetrahydrocannabinol (THC) are two phytocannabinoids that have been shown to reduce the growth of glioma xenographs in rats and mice [62]. When encapsulated in PCL microspheres, a 64 and 79\% release of CBD and THC was achieved, respectively, after five days followed by a sustained release through day 13 [62].

3.5. Endogenous inhibitors, bone morphogenetic protein-7, interleukin-2, and recombinant adenovirus

Endogenous inhibitors such as hemopexin (PEX) and platelet factor 4 fragment ( $\mathrm{PF}-4 / \mathrm{CTF}$ ) are angiogenesis inhibitors that have the potential to be applied for local glioma treatment. PEX ( $29 \mathrm{kDa}$ ) inhibits tumor cell proliferation, migration, and invasion by regulating a critical step controlling endothelial cell invasion while PF-4/CTF (2.7 kDa) 
directly associates with and alters the conformation of angiogenic factors which inhibits endothelial cell proliferation and tumor growth [45]. Benny et al. has investigated PEX and PF-4/CTF loaded separately in PLGA microspheres and their in vitro release profiles [45,46]. The proteins were shown to have a sustained release for 30 days [45], with PF$4 / \mathrm{CTF}$ released faster due to its lower molecular weight. Endothelial and glioblastoma cell proliferation assays were conducted to test the bioactivity of these proteins after encapsulation. PF-4/CTF inhibited HUVEC proliferation by 33 to $47 \%$ over 6 days and inhibited BCE proliferation by 42 to $54 \%$ over 11 days. PEX inhibited HUVEC proliferation by 36 to $62 \%$ over 10 days and inhibited U87-MG glioma line by 29 to $65 \%$ over 6 days. Additionally, release of rhodamine labeled PF-4/CTF from PLGA with an "uncapped" free carboxyl acid group and a "capped" esterified end group was investigated [46]. While both PLGA carriers demonstrated an initial burst of $10 \%$, the uncapped PLGA released over 17 days in contrast to the capped, more hydrophobic PLGA that had a continuous release over 30 days.

Bone morphogenetic protein-7 (BMP-7) is a growth factor that can act as a suppressor for glioblastoma initiating cells responsible for tumor re-initiation and sustained growth [67]. Reguera-Nuñez et al. encapsulated the nanocomplex of heparin-BMP-7 coated with Tetronic ${ }^{\circledR}$ in PLGA microspheres and demonstrated a triphasic release profile of BMP-7: a burst release less than 3\% followed by a 15 day slow release and then by a sustained released through day 60 [67]. When tested in vitro, BMP-7 was found to maintain its bioactivity against U-87MG cells even after 90 days of release.

Interleukin-2 is a cytokine with immunomodulatory activity, producing a strong, local inflammatory response adjacent to potential antigens. In addition, it enhances natural killer/leukocyte-activated killer cell activity [68]. Interleukin-2 encapsulated in gelatin/chondroitin6-sulfate microspheres was studied by Rhines et al. for a localized delivery to the brain [68]. The typical release was characterized by a high release rate during the first 7 days followed by a slower, sustained release for 6 weeks.

An adenovirus-mediated transfer of cytotoxic gene products also has potential for the treatment of brain tumors [69]. However, the immune response following adenovirus administration prevents re-dosing due to the production of neutralizing anti-adenoviral antibodies. Therefore, a delivery vehicle that diminishes immune response and delivers locally is necessary. PLGA microspheres encapsulating recombinant adenovirus was reported by Beer et al. in an attempt to reduce this immunogenicity [69]. The release of the virus was demonstrated to be dependent on the virus input volume: as the input volume of the aqueous virus solution was increased from 50 to $150 \mu \mathrm{L}$, the release rate increased accordingly. Over a $200 \mathrm{~h}$ time period, the highest input volume resulted in about $45 \%$ release while the lowest input volume demonstrated approximately $10 \%$ release. The higher release rate could be a result of the heterogeneity in particle shape and size that was reported for higher input volumes while the lower input volume resulted in more uniform particles. Utilizing 9 L gliosarcoma cells, a dose dependent response in cell survival showed that the recombinant adenovirus maintained its capability to transduce cells and be an effective therapy for gliomas.

\section{In vivo studies}

The following sections review the in vivo studies of various chemotherapeutic encapsulated polymeric microspheres for localized glioma therapy. When preclinical studies demonstrate significant potential (high encapsulation efficiency, sustained and tunable release, drug bioactivity, etc.) it is appropriate to bring the therapy to early stage clinical evaluation. The effectiveness, as measured by tumor volume reductions and life expectancy, will be highlighted in describing the type of study performed.

\subsection{Animal studies}

\subsubsection{Paclitaxel}

The effectiveness of paclitaxel in vivo has been studied utilizing mice models with induced 9 L gliosarcoma or C6 glioma tumors [58-60,70]. Naraharisetti et al. implanted paclitaxel loaded PLGA microspheres formulated by either spray drying or the EHDA method along the tumor sites of BALB/c nude mice bearing C6 glioma cells subcutaneously [59]. The microspheres formulated by spray drying were able to arrest tumor growth for more than 45 days when applied 6 days after tumor cell inoculation. However, when applied at day 9, the tumor did not respond to treatment due to the large tumor size. The EHDA generated microparticles were applied 14 days after tumor cell inoculation (larger tumor volume) and arrested tumor growth for 10 days after the first injection. However, tumor growth rate increased after a repeated injection two weeks later of the EHDA paclitaxel loaded PLGA microparticles. Overall, during 21 days of treatment, tumor growth was inhibited by $59 \%$ and $65 \%$ for the spray dried and EHDA microparticles, respectively, in comparison to the blank placebo; however, over longer time points, the spray-dried formulated, slower releasing microspheres were the most effective, an example of preliminary in vitro studies aiding in the interpretation of animal studies. Similarly, Ranganath et al. demonstrated the best results for paclitaxel encapsulated PLGA microspheres in alginate beads by reducing tumor volume $85 \%$ and $78 \%$ compared to a blank control and the free drug form of paclitaxel (Taxol), respectively, after 21 days of treatment [58]. As opposed to Naraharisetti et al., Ranganath et al. implanted the drug loaded beads into the tumor itself instead of peripherally at the tumor site.

Paclitaxel encapsulated polilactofate microspheres were also tested against the $9 \mathrm{~L}$ gliosarcomas cells in Fischer 344 rats as reported by Li et al. [60]. In this study, drug encapsulated microspheres were compressed in a wafer in the presence of PEG and implanted intracranially, on top of the existing tumor. Biocompatibility studies showed that the polilactofate implants' immune response was comparable to the clinically used poly(carboxyphenoxypropane-sebacic acid) (PCPP-SA) GLIADEL $®$ wafers and did not produce additional overt toxicity. These paclitaxel implants also doubled the median survival of the rats in comparison to the control and produced long-term survivors at day 90 when the study was ended. The same dosage of paclitaxel applied systemically had no effect on survival, demonstrating the potential for a localized, sustained release of this drug against gliomas. These wafers were also tested in combination with radiation treatment [70]. When the paclitaxel wafers were implanted and followed five days later with a 20 Gy external beam single-dose radiotherapy, 8 out of 13 animals survived the study and median survival was not reached (greater than 150 days). This high survival is attributed to paclitaxel acting as a radiosensitizer. Only 3 animals survived with a median survival of 45 days when radiation therapy was applied first, followed by the paclitaxel wafer administration.

This same polilactofate system (named Paclimer ${ }^{\circledR}$ ) was tested in a higher animal model to determine its potential for clinical application by Pradilla et al. [39]. In a canine dose escalation toxicity study, it was concluded that Paclimer ${ }^{\circledR}$ is safe to be intracranially delivered, as evidenced by the lack of systemic toxicity and myelosuppresion at two different dosages: $2 \mathrm{mg} / \mathrm{kg}$ and $20 \mathrm{mg} / \mathrm{kg}$. Adverse effects that did occur included wound infections and a brain abscess, both of which responded to antibiotic therapy.

\subsubsection{5-Fluorouracil}

The radiosensitizer 5-fluorouracil has been extensively used for in vivo studies, progressing to the point of a Phase II clinical trial [25, 31-37]. 5-FU encapsulated PMM 2.1.2 microspheres were tested by Fournier et al. against the F98 tumor cell line inoculated intracranially in Fisher rats [35]. It was found that the 5-FU encapsulated microspheres increased the median survival to $34.1 \pm 5.6$ days compared to 

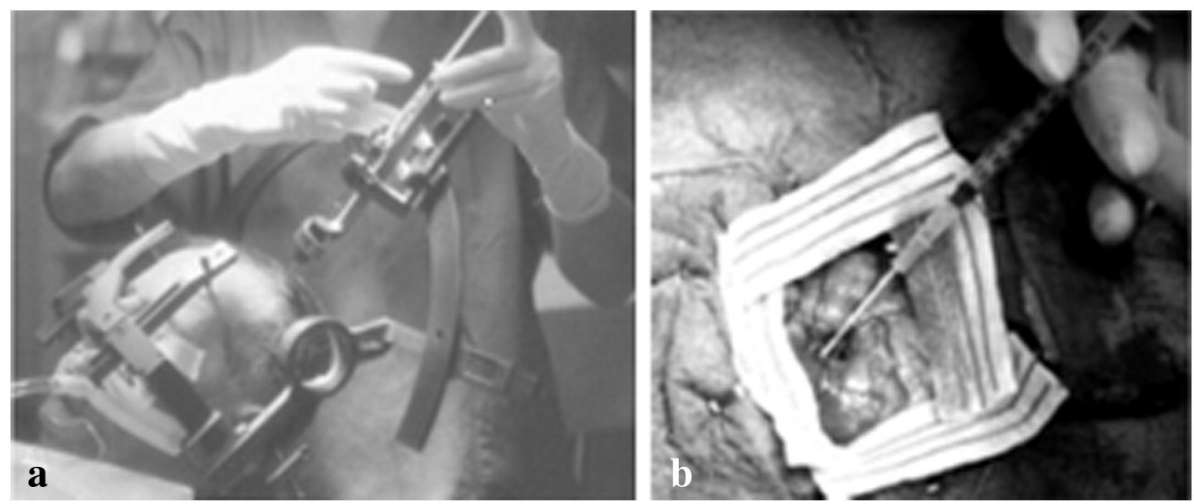

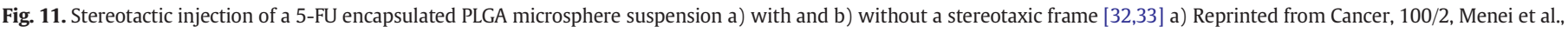

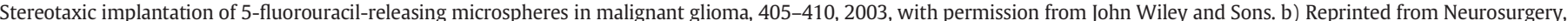

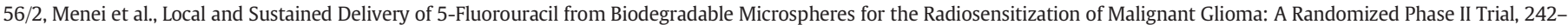
247, 2003, with permission from Wolters Kluwer Health.

the no treatment control at $22.7 \pm 3.3$ days and a 5 -FU solution applied stereotactically at $26.5 \pm 2.4$ days.

The most common system for 5-FU encapsulated microspheres applied to gliomas consists of stereotactically injected, 5-FU loaded PLGA microspheres as shown in Fig. 11 [25,31-34,36,37]. Menei et al. conducted an initial intracranial study using 5-FU loaded PLGA microspheres against a C6 glioma cell line on Sistar and Sprague-Dawley rats [31]. For this study, two formulations with different release rates, fast releasing ( $100 \%$ release in $72 \mathrm{~h}$ ) and slow releasing $(100 \%$ in 18 days) were used without clinical or histological signs of neurological toxicity. When tested against the tumor cell line, the slow release group significantly decreased mortality. The fast releasing group was not included due to a low sampling number and stringent analysis criterion. Intratumoral injection of 5-FU solution did not modify mortality when compared to the untreated control group.

Lemaire et al. found that the stereotactic administration of the 5-FU loaded PLGA microspheres slowed tumor development by a factor of three when compared to a bolus 5-FU solution, intratumorally injected, for intracranial C6 glioma cells in Sprague-Dawley rats [25]. Overall, both protocols increased survival time by $50 \%$ over the controls. However, overall survival was not significantly different between the two therapies, with a $30 \pm 1$ day and $32 \pm 2$ days survival for the bolus and microsphere injection, respectively. An interesting result of this experiment is that tumor proliferation was significantly reduced in the vicinity of the stereotactic injection site before regrowth. This led to the need for multi-injection sites protocols, further elucidated in a Sprague-Dawley rat study using the same system with $\left[{ }^{3} \mathrm{H}\right] 5-\mathrm{FU}$ to monitor diffusion [36]. In the tumor bearing rats, the drug encapsulated microspheres were found to diffuse $\sim 1.5 \mathrm{~mm}$ distance from the injection site and to release 5-FU at a maximum of $3 \mathrm{~mm}$. Multiple injections localized over the whole area of the tumor would be needed for the best chance of tumor eradication.

Additional studies on 5-FU encapsulated PLGA microspheres by Roullin et al. utilized its radiosensitizing potential and applied 36 Grays (Gy) to test therapeutic potential in C6 glioma bearing Sprague-Dawley rats [37]. Two drug loaded microsphere formulations characterized by a fast ( 7 days for $100 \%$ release) and slow release (20 days for $100 \%$ release) were explored. The 5-FU loaded microspheres in combination with radiotherapy caused a $47 \%$ complete remission rate, while radiotherapy alone and no therapy had $8 \%$ and $0 \%$ remission, respectively. The survival for the slow and fast releasing drug loaded microspheres in combination with radiotherapy was $57 \%$ and $41 \%$, respectively, while survival without the radiotherapy was 0 and $27 \%$, respectively.

\subsubsection{Carboplatin and $B C N U$}

PLGA is a widely used carrier for encapsulating the chemotherapeutics carboplatin and BCNU for in vivo studies [40-42,53]. In a study against RG2 cells in male Fisher rats, Emerich et al. showed that a significant retention of carboplatin in the brain tissue was detected after a local implantation of drug loaded microspheres when compared to a bolus dose [41]. The bolus dose resulted in a carboplatin tissue level decrease of $>99 \%$ after three days, significantly greater than the microsphere dose decrease of $44 \%$. It was also found that the location of microsphere injection played an important role in survival. The rats that received drug loaded microspheres through direct injection into the tumor cavity did not show a significant improvement when compared to the control animals that underwent a tumor resection alone. Direct injection of the highest dose (100 $\mu$ g carboplatin) increased median survival from 20 days (no treatment) to 36 days while tumor resection alone increased median survival to 30 days. However, animals that were injected along the perimeter of the tumor cavity with carboplatin loaded PLGA microspheres had a higher survival compared to the controls. The $100 \mu \mathrm{g}$ of encapsulated carboplatin applied along the perimeter increased median survival by $110 \%$ with two animals surviving the 180 day duration of the experiment. Additionally, a significant decrease in tumor volume was detected for the perimeter injection when compared to resection alone: $14.8 \mathrm{~mm}^{3}$ compared to $99.5 \mathrm{~mm}^{3}$ at day 28 and $50.7 \mathrm{~mm}^{3}$ versus $99.5 \mathrm{~mm}^{3}$ at day 38 , respectively. Similar studies were conducted by the same group testing BCNU loaded PLGA microspheres injected along or inside the tumor cavity [41]. The results supported the trend demonstrated with the carboplatin loaded PLGA microspheres. PLGA microspheres with $100 \mu \mathrm{g}$ of encapsulated BCNU injected directly into the tumor cavity had a lower median survival than microspheres injected along the tumor perimeter; a $36 \%$ and $61 \%$ increase, respectively, compared to tumor resection alone.

These promising results led to a second study by Emerich et al., this time testing the system against inoperable brain tumors in male Fisher rates inoculated with the RG2 cell line [42]. In the first part of the study, relatively small, three day old tumors were injected with 10,50 , and $100 \mu \mathrm{g}$ of carboplatin encapsulated in PLGA in the center of the tumor. Median survival was dose dependent, showing an increase of $155 \%$ and $178 \%$ for 50 and $100 \mu \mathrm{g}$, respectively, but with no impact at the lowest dosage. A bolus injection of the highest dosage increased median survival by only $33 \%$, showcasing the efficacy of sustained release. In the second part of the study, the tumors were allowed to grow for 8 days and the efficacy of perimeter injected versus center injected carboplatin-PLGA microspheres was tested. An increase of $39 \%$ in 
median survival for intratumorally injected, drug loaded particles $(100 \mu \mathrm{g})$ was detected versus $189 \%$ for peritumoral injection, again supporting the efficacy of perimeter injection. A similar tendency was found for BCNU loaded PLGA microspheres: an increase of $25 \%$ versus $105 \%$ for intratumoral and peritumoral injections, respectively. The same dosage applied peritumorally as a bolus injection increased median survival by only $25 \%$ [42].

These studies provided evidence for an increased efficacy of the drug encapsulated microspheres if applied peritumorally. For this reason, Emerich et al. conducted another study to determine the effect of carboplatin dosage and the best location for peritumoral injections [53]. Two different spatial injection patterns were used, one with 4 injection sites spaced $4 \mathrm{~mm}$ apart and a second one with 8 sites spaced $2 \mathrm{~mm}$ apart. The first method produced no survival benefit when compared to the median survival of tumor resection. However, the 8 injection sites increased median survival by $141 \%$. Interestingly, if 8 sites are injected on only one side of the tumor cavity, survival was not enhanced. A dosing limit was also found with a $200 \mu \mathrm{g}$ encapsulated carboplatin injection resulting in lower survival rates than the control with no treatment, most likely due to central nervous system toxicity.

In a different study, Emerich et al. elucidated the effects of repeated peritumoral injection of carboplatin-PLGA microspheres [40]. Carboplatin loaded particles were injected into 8 sites around the RG2 tumor resection cavity. This initial therapy increased median survival by $141 \%$ over tumor debulking. However, when the same therapy was repeated 20 days later, median survival increased to $219 \%$. One third of the rats studied were long term survivors, greater than 150 days. Histology demonstrated the complete eradication of the tumor for the double injection. The next step taken was to mix both $\mathrm{BCNU}$ and carboplatin encapsulated microspheres together and inject peritumorally. As shown in Fig. 12, when mixed together and injected into 8 sites, an increase in median survival compared to tumor debulking was $193-245 \%$. However, when the drugs were injected separately but spatially alternating around the tumor site, an increase of only 55-77\% resulted. This highlights the idea that a polypharmacy therapy could further improve survival beyond the traditional application of a single chemotherapeutic.

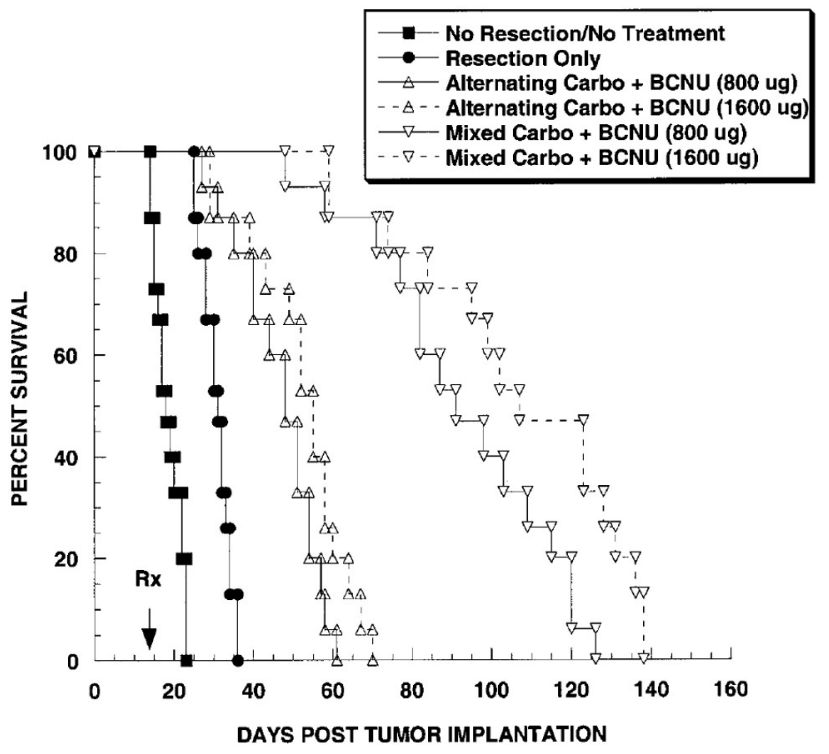

Fig. 12. The effect of combining carboplatin and BCNU encapsulated PLGA microspheres on survival. Microspheres were either injected separately but spatially alternating around the tumor site or mixed together and delivered as a single suspension after tumor resection [40] Reprinted from Cell Transplantation, 11/1, Emerich et al., Injection of Chemotherapeutic Microspheres and Glioma IV: Eradicating Tumors in Rats, 47-54, 2002, with permission from Cognizant Communication Corporation.

\subsubsection{Other small molecule chemotherapeutics}

Camptothecin is another chemotherapeutic that was tested in vivo against gliomas using PLGA microspheres as the drug delivery vehicle $[49,63,71]$. Ozeki et al. injected camptothecin encapsulated PLGA microspheres suspended in a TGP directly in the brain of SpragueDawley rats against C6 glioma cells [49]. At physiological temperature, the TGP underwent a phase transition from a liquid to a gel, holding the microspheres at the tumor area. Localized treatment with camptothecin-PLGA in the TGP increased survival significantly, with one third of the rats surviving more than 60 days compared to 16 days of mean survival for animals with no treatment. The same dosage of free camptothecin dispersed in TGP had a median survival of only 21 days. A follow up study was conducted, this time with the drug delivery system injected after tumor resection [71]. Tumor resection led to a median survival of 18.5 days while resection plus injection of camptothecin-PLGA in the TGP resulted in a 24 and 26 day median survival for a 10 and $30 \mu \mathrm{g}$ camptothecin dosage, respectively. The camptothecin-PLGA in the TGP was also applied without tumor resection, resulting in the same median survival. However, only groups with both tumor resection and the drug delivery system resulted in long-term survivors.

Using the same PLGA microspheres in the TGP, Ozeki et al. compared the efficacy of encapsulated camptothecin and vincristine on the in vivo survival of male Sprague-Dawley rats intracranially inoculated with C6 glioma cells [63]. As shown in Table 1, camptothecin encapsulated microspheres dispersed in TGP increased survival in a dosage dependent manner, 24 and 26 days for drug dosages of 10 and $30 \mu \mathrm{g}$, respectively, compared to the untreated median survival of 18 days. Vincristine encapsulated PLGA microspheres in TGP had a 28, 33, and 33 days median survival for 1,3 , and $10 \mu \mathrm{g}$ drug dosage, respectively. Unlike the camptothecin group, there were several long term survivals in the vincristine groups, indicating that vincristine is more efficient than camptothecin in this delivery system against C6 glioma cells. A possible reason for this is the longer, extended release of vincristine from PLGA compared to camptothecin. In addition, the authors state that it has been reported that vincristine is more cytotoxic against this particular glioma line than camptothecin [63].

Temozolomide encapsulated PLGA microspheres were also utilized against the Sprague-Dawley rats intracranially inoculated with the C6 glioma cell line $[28,72]$. Zhang et al. reported that animals without treatment or on a 5-day oral temozolomide treatment had a median survival of 20 and 27 days, respectively, while median survival for a group that received PLGA encapsulated temozolomide through direct implantation on the tumor surface increased to 46.5 days [72]. Tumor volume was shown to be reduced by $56.6 \%$ when compared to the no treatment control.

Zhang et al. also studied temozolomide encapsulated PLGA microspheres with the addition of orally delivered vatalanib, an antiangiogenesis drug [28]. The combination of temozolomide loaded PLGA microspheres and orally administrated vatalanib increased median survival to 40.5 days, while the non-treatment group, the

\section{Table 1}

The effect of vincristine (VCR) and camptothecin (CPT) encapsulated PLGA microspheres dispersed in a TGP on median survival, ${ }^{*} \mathrm{p}<0.05,{ }^{* *} \mathrm{p}<0.01,{ }^{* * *} \mathrm{p}<0.005$ [63] Reprinted from International Journal of Pharmaceutics, 427, Ozeki et al., Improvement of survival in C6 rat glioma model by a sustained drug release from localized PLGA microspheres in a thermoreversible hydrogel, 299-304, 2012, with permission from Elsevier.

\begin{tabular}{lll}
\hline Treatment & $\begin{array}{l}\text { Median survival } \\
\text { period (days) }\end{array}$ & $\begin{array}{l}\text { Number of long-term } \\
\text { survivors }(>60 \text { days })\end{array}$ \\
\hline Non-treatment (control) & 18 & $0 / 7$ \\
VCR $(10 \mu \mathrm{g}) /$ PLGA/PBS & $23.5^{* * *}$ & $0 / 6$ \\
VCR $(1 \mu \mathrm{g}) /$ PLGA/TGP & $28^{* * *}$ & $2 / 6$ \\
VCR $(3 \mu \mathrm{g}) /$ PLGA/TGP & $33^{* * *}$ & $2 / 7$ \\
VCR $(10 \mu \mathrm{g}) /$ PLGA/TGP & $33^{* * *}$ & $0 / 6$ \\
CPT $(10 \mu \mathrm{g}) /$ PLGA/TGP & $24^{*}$ & $0 / 5$ \\
CPT $(30 \mu \mathrm{g}) /$ PLGA/TGP & $26^{* *}$ & $0 / 5$
\end{tabular}


group that received drug encapsulated microspheres alone, and the group with orally delivered vatalanib alone showed a median survival of 20,37 , and 23.5 days, respectively. Additionally, the combination therapy reduced tumor volume by $74.4 \%$ while a volume reduction of $68 \%$ and $28.1 \%$ was detected for temozolomide-PLGA and oral vatalanib, respectively. These results show the promise of therapies that use multiple chemotherapeutics that attack different tumorigenic pathways.

Temozolomide was also encapsulated in PCPP-SA microspheres and tested in vivo by Dong et al. [73]. For athymic mice with the human glioma cell line SHG44 inoculated subcutaneously, the tumor weight was significantly reduced to $350 \mathrm{mg}$ for direct injection of encapsulated temozolomide compared to $600 \mathrm{mg}$ tumor weight for oral temozolomide and $825 \mathrm{mg}$ tumor weight for blank microspheres. When tested intracranially, tumor volume was also reduced when the polymeric microsphere delivery was compared to the oral treatment, approximately 5 and $15 \mathrm{~mm}^{3}$, respectively, with a tumor volume of $35 \mathrm{~mm}^{3}$ for the blank microspheres control.

The small molecule imatinib mesylate was encapsulated by Benny et al. in PLGA microspheres for a localized, sustained release and tested in vivo, both subcutaneously and intracranially [47]. Swiss nude and C57/BL mice were subcutaneously inoculated with human U87-MG and GL261 glioblastoma cells, respectively. Tumor volume was suppressed by $79 \%$ after 30 days and $88 \%$ after 22 days for the GL261 and U87-MG cell line, respectively. The U87-MG cell line was then tested intracranially in Swiss nude mice. It was demonstrated that the imatinib mesylate encapsulated PLGA microspheres significantly reduced tumor growth by $79 \%$ when compared to blank microspheres 14 days after intratumoral injection.

The effect of applying mitoxantrone encapsulated PLGA microspheres against intracranially inoculated RG2 cells in Fisher rats was demonstrated by Yemisci et al. [52]. The time point of the drug delivery system application was studied, with the treatment occurring either simultaneously with tumor cell inoculation or seven days after tumor growth. It was found that in rats treated with mitoxantrone-PLGA injected 7 days after tumor inoculation, tumor volumes were $17 \pm 4$ and $23 \pm 2 \mathrm{~mm}^{3}$ on days 15 and 35 , respectively, indicating a reduction of 77.6 and $78.5 \%$ when compared to untreated rats. However, when the microspheres and cells were injected simultaneously, no tumor formation was reported.

The idea of a "drug cocktail" or multiple chemotherapeutics applied together was studied by Allhenn et al. using celecoxib, etoposide, and elacridar encapsulated PLGA microspheres that released over seven days after a $85 \%$ (celecoxib, elacridar) and 35\% (etoposide) burst release [74]. When tested against a subcutaneous F98 tumor in male Fischer F344 rats, an increase in mean survival was seen for all microsphere groups tested including celecoxib microspheres, etoposide microspheres, combined etoposide and celecoxib microspheres, and combined etoposide, celecoxib, and elacridar microspheres. However, the mean survival was only prolonged significantly for etoposide microspheres and the combination of etoposide and celecoxib microspheres when compared to the blank microsphere control. The low impact of the combinational therapy was attributed to the small diffusion of the drugs from the microspheres into the tumor tissue [74].

\subsubsection{Endogenous inhibitors and interleukin-2}

Protein-based chemotherapeutics have also been encapsulated in PLGA microspheres and studied in vivo for efficacy against brain tumors. For example, Benny et al. studied the C-terminal fragment of platelet factor 4, an anti-angiogenic agent, encapsulated in PLGA microspheres against intracranially inoculated U87-MG in Swiss nude mice [46]. Fourteen days after microsphere injection, a significant reduction of $72 \%$ in the tumor volume was measured when compared to blank microspheres, correlating with a four-fold increase in tumor cell apoptosis relative to the control.
Another protein, interleukin-2, was encapsulated in gelatin/ chondroitin-6-sulfate microspheres and tested in vivo $[68,75,76]$. Hanes et al. tested interleukin-2 loaded microspheres injected simultaneously with intracranially inoculated B16-F10 melanoma cells in C57/ B16 mice and $9 \mathrm{~L}$ gliosarcoma cell lines in Fisher rats. These particular cell lines are characterized by poor immunogenicity, making B16-F10 and C57/B16 good models for testing interleukin-2 [75]. In the B16F10 animal model, treatment with blank microspheres or with cells that secrete interleukin-2 had a median survival of 18 and 29 days, respectively, while treatment with drug loaded microspheres significantly enhanced survival, with 16 of 18 mice surviving greater than 75 days. To test the development of anti-tumor immunological memory from this treatment, the surviving rats were re-inoculated with B16 cells in the contralateral hemisphere of the brain. Five out of 12 rats survived without further treatment, demonstrating development of immunological memory, compared to the control group without previous treatment that all died by day 20 . Similarly, a significant increase in median survival was also detected for the $9 \mathrm{~L}$ cell line animal model treated with the interleukin-2 encapsulated microspheres; 3 of 8 animals survived longer than 100 days while all animals in the blank microsphere group died by day 31 [75].

A further study using the interleukin-2 encapsulated gelatin/ chondroitin-6-sulfate microspheres was conducted by Rhines et al., evaluating the efficacy of combining the interleukin-2 microspheres with the chemotherapeutic BCNU, applied as polymeric wafers [68]. For 9 L wild-type cells inoculated intracerebrally in Fischer rats, a median survival of 18 days was observed with the application of blank polymers. The application of mono-therapy increased survival: injected interleukin-2 microspheres increased median survival to 24 days and implanted $3.8 \%$ and $10 \%$ loaded BCNU wafers to 24 and 32.5 days, respectively. However, combination therapy had a greater survival, with interlukin-2 microspheres and 3.8\% BCNU achieving a median survival of 28.5 days and one long term survivor while an increase to $10 \% \mathrm{BCNU}$ with the microspheres had a 45.5 day median survival with $25 \%$ of the group being long-term survivors. This study continues to support the concept of synergistic benefit by using two pharmacologic strategies, an immunotherapy and a chemotherapy.

A similar approach was taken by Hsu et al. to study the benefits of combining interleukin-2 in gelatin/chondroitin-6-sulfate microspheres with adriamycin, an anthracycline antibiotic that blocks DNA and RNA synthesis by inhibiting topoisomerase II, applied as a polymer wafer [76]. When applied to a $9 \mathrm{~L}$ intracranial tumor in Fisher rats, the mono-therapies of adriamycin wafers and interleukin-2 microspheres increased media survival to 30 and 39 days, respectively, compared to the 18 day median survival for the untreated group. The combination of the two drugs resulted in a median survival of 53 days, a significant increase from the singular treatments.

\subsection{Clinical trials}

Based on the decrease in mortality, lack of toxicity, and sustained drug delivery demonstrated by the 5-FU encapsulated PLGA microspheres in a murine model, a pilot study with eight patients with newly diagnosed glioblastoma was conducted by Menei et al. to further elucidate the potential of this microsphere drug delivery system [34]. The drug loaded microspheres were applied along the walls of the tumor resection cavity for total dosages of either 70 or $132 \mathrm{mg}$. External beam radiotherapy was applied between the second and seventh day after surgery for a total of $59.4 \mathrm{~Gy}$. The dose of $132 \mathrm{mg}$ caused recurrent brain swelling 3 weeks after radiotherapy and required steroid treatment before completing the radiation. It was found that significant levels of 5-FU were present in the cerebral spinal fluid one month after implantation, enabling optimal radiosensitization. All patients treated with the 5-FU-PLGA system had a median survival time of 98 weeks at the last evaluation, greater that the 50.6 weeks for resection 
and radiotherapy alone. The higher dosage had two long term survivors that were in disease remission at 139 and 153 weeks.

To further confirm the potential of the 5-FU encapsulated PLGA microspheres against glioma cells, a Phase I study was conducted by Menei et al. [33]. This study included 10 patients with newly diagnosed, inoperable, malignant gliomas that were dosed one time with $132 \mathrm{mg}$ of encapsulated 5-FU. Different stereotactical injection trajectories (one to seven deposits) were applied dependent on the 3-D conformation of the tumor. The overall median survival was 40 weeks with 2 long-term survivors ( 71 and 89 weeks). Based on the aforementioned promising results, a Phase II trial was conducted [32]. This trial had two treatments groups: in Arm A, 49 patients receiving 5-FU releasing microspheres (130 mg) with early radiotherapy (59.4 Gy) and in Arm B, 46 patients with only early radiotherapy $(59.4 \mathrm{~Gy})$. All therapies were applied after gross tumor resection. A median overall survival of 15.2 months was achieved in Arm A, approximately 2.9 months longer than in Arm B. However, it did not reach statistical significance. Time to tumor progression was also not significantly different, 6.5 months in Arm A and 6.3 months in Arm B. While this Phase II study shows some potential, it was not designed and powered sufficiently to show a statistically significant result.

\section{Conclusions}

Malignant brain tumors present a formidable challenge. The treatment must be effective and also provide an acceptable quality of life for the patient. The blood brain barrier prevents the majority of chemotherapeutics from reaching the brain and the few that do penetrate via systemic administration dose the entire body, not just the brain tumor. The approach of a localized application of degradable, polymeric microspheres with encapsulated drug has great potential for overcoming the challenge of the blood brain barrier while minimizing the severe side effects of chemotherapy. As reviewed here, polymeric microspheres provide a versatile platform for specifically tailoring drug loading and release kinetics by tuning the formulation parameters. This ability to precisely create and tailor drug delivery systems provides a more personalized medicine approach. For example, the polymer type, release kinetics, and drug dosage could be matched to the tumor type, grade, and size to provide greatest impact. Also, the physician could have microsphere formulations, each loaded with different drugs. Thus, the physician could customize the therapy for each patient based on choice of drug, or using mixtures of microspheres, each with different drugs.

The delivery site can also play an important role in the therapeutic effectiveness of microsphere drug delivery systems. As discussed in the in vivo studies section, peritumoral injections of drug encapsulated microspheres resulted in a longer median survival than intratumoral injection. The mechanism for this enhanced action of peritumoral delivery are unclear and therefore, this concept needs to be further studied to determine the best injection pattern for specific drugs as each will diffuse differently through the brain tissue.

In addition, research into the polypharmacy approach with "synergistic" benefits from multiple drugs delivered simultaneously or staged temporally is a promising avenue for future study. The resilient glioma tumors can be attacked with multiple chemotherapeutics, targeting different tumorigenic pathways over a sustained period of time and suppress tumor growth if not eradicate the tumor itself. Many polypharmacy studies have shown enhanced efficacy over administration of single drugs. Polypharmacy also intersects with a personalized medicine approach, as the physician can choose the drug combination that would be most beneficial based on the tumor diagnosis.

Drug encapsulated, degradable, polymeric microspheres are a straightforward platform for providing a wide array of treatment options that, if fully realized, can significantly and beneficially impact the treatment of brain tumors. Benefits include lower total doses due localized delivery, sustained release, regulatory agency acceptance of microsphere delivery modes, and the ability to delivery combinations of different drugs. Given the potential for success suggested by animal studies and the dire prognosis associated with glioblastoma multiforme it is surprising how relatively few clinical studies have used injected or implanted drug-loaded microspheres. The authors hope this review article will stimulate increased interest in this promising therapeutic option.

\section{Acknowledgments}

Support has been received from the NSF Graduate Research Fellowship under Grant No. DGE-0718124 to J. Alaina Floyd and from the Michael and Myrna Darland Endowment to the Department of Bioengineering, University of Washington.

\section{References}

[1] N. Howlader, N. A., M. Krapcho, N. Neyman, R. Aminou, S.F. Altekruse, C.L. Kosary, J. Ruhl, Z. Tatalovich, H. Cho, A. Mariotto, M.P. Eisner, D.R. Lewis, H.S. Chen, E.J. Feuer, K.A. Cronin (Eds.),SEER Cancer Statistics Review, 1975-2009 (Vintage 2009 Populations), April 2012 (http://seer.cancer.gov/csr/1975_2009_pops09/</csr/ 1975_2009_pops09/>).

[2] Society A.C., Cancer Fact and Figures 2012, 2012.

[3] E.C. Holland, Glioblastoma multiforme: the terminator, Proc. Natl. Acad. Sci. U. S. A. 97 (12) (2000) 6242-6244.

[4] K. Anton, J.M. Baehring, T. Mayer, Glioblastoma multiforme: overview of current treatment and future perspectives, Hematol. Oncol. Clin. North Am. 26 (4) (2012) 825-853.

[5] E.G. Van Meir, C.G. Hadjipanayis, A.D. Norden, H.K. Shu, P.Y. Wen, J.J. Olson, Exciting new advances in neuro-oncology the avenue to a cure for malignant glioma, CA Cancer J. Clin. 60 (3) (2010) 166-193.

[6] C. Buonerba, G. Di Lorenzo, A. Marinelli, P. Federico, G. Palmieri, M. Imbimbo, P. Conti, G. Peluso, S. De Placido, J.H. Sampson, A comprehensive outlook on intracerebral therapy of malignant gliomas, Crit. Rev. Oncol. Hematol. 80 (1) (2011) 54-68.

[7] M.I. Alam, S. Beg, A. Samad, S. Baboota, K. Kohli, J. Ali, A. Ahuja, M. Akbar, Strategy for effective brain drug delivery, Eur. J. Pharm. Sci. 40 (5) (2010) 385-403.

[8] D.R. Groothuis, The blood-brain and blood-tumor barriers: a review of strategies for increasing drug delivery, Neuro Oncol. 2 (1) (2000) 45-59.

[9] G. Orive, O.A. Ali, E. Anitua, J.L. Pedraz, D.F. Emerich, Biomaterial-based technologies for brain anti-cancer therapeutics and imaging, Biochim. Biophys. Acta Rev. Cancer 1806 (1) (2010) 96-107.

[10] F.H. Hochberg, A. Pruitt, Assumptions in the radiotherapy of glioblastoma, Neurology 30 (9) (1980) 907-911.

[11] A.J. Sawyer, J.M. Piepmeier, W.M. Saltzman, New methods for direct delivery of chemotherapy for treating brain tumors, Yale J. Biol. Med. 79 (3-4) (2006) 141-152.

[12] C. Guerin, A. Olivi, J.D. Weingart, H.C. Lawson, H. Brem, Recent advances in brain tumor therapy: local intracerebral drug delivery by polymers, Investig. New Drugs 22 (1) (2004) 27-37.

[13] W.B. Dang, T. Daviau, P. Ying, Y. Zhao, D. Nowotnik, C.S. Clow, B. Tyler, H. Brem, Effects of GLIADEL(R) wafer initial molecular weight on the erosion of wafer and release of BCNU, J. Control. Release 42 (1) (1996) 83-92.

[14] M. Westphal, D.C. Hilt, E. Bortey, P. Delavault, R. Olivares, P.C. Warnke, I.R. Whittle, J. Jaaskelainen, Z. Ram, A phase 3 trial of local chemotherapy with biodegradable carmustine (BCNU) wafers (Gliadel wafers) in patients with primary malignant glioma, Neuro Oncol. 5 (2) (2003) 79-88.

[15] G. Tiwari, R. Tiwari, B. Sriwastawa, L. Bhati, S. Pandey, P. Pandey, S.K. Bannerjee, Drug delivery systems: an updated review, Int. J. Pharm. Investig. 2 (1) (2012) 2-11.

[16] E. Nance, C. Zhang, T.Y. Shih, Q.G. Xu, B.S. Schuster, J. Hanes, Brain-penetrating nanoparticles improve paclitaxel efficacy in malignant glioma following local administration, ACS Nano 8 (10) (2014) 10655-10664.

[17] J.B. Zhou, T.R. Patel, R.W. Sirianni, G. Strohbehn, M.Q. Zheng, N. Duong, T. Schafbauer, A.J. Huttner, Y.Y. Huang, R.E. Carson, Y. Zhang, D.J. Sullivan, J.M. Piepmeier, W.M. Saltzman, Highly penetrative, drug-loaded nanocarriers improve treatment of glioblastoma, Proc. Natl. Acad. Sci. U. S. A. 110 (29) (2013) 11751-11756.

[18] V.J.C. Mohanraj, Y., Nanoparticles-a review, Trop. J. Pharm. Res. 5 (1) (2006) 561-573.

[19] S. Freiberg, X. Zhu, Polymer microspheres for controlled drug release, Int. J. Pharm. $282(1-2)(2004) 1-18$

[20] U. Edlund, A.C. Albertsson, Degradable polymer microspheres for controlled drug delivery, Degradable Aliphatic Polyesters, 157 2002, pp. 67-112.

[21] J.H. Park, M.L. Ye, K. Park, Biodegradable polymers for microencapsulation of drugs, Molecules 10 (1) (2005) 146-161.

[22] C. Vilos, L.A. Velasquez, Therapeutic strategies based on polymeric microparticles, J. Biomed. Biotechnol. 2012 (2012) Article ID 672760

[23] L. Hu, H. Zhang, W. Song, An overview of preparation and evaluation sustained-release injectable microspheres, J. Microencapsul. 30 (4) (2013) 369-382.

[24] V.T. Tran, J.P. Benoit, M.C. Venier-Julienne, Why and how to prepare biodegradable, monodispersed, polymeric microparticles in the field of pharmacy? Int. J. Pharm. $407(1-2)$ (2011) 1-11.

[25] L. Lemaire, V.G. Roullin, F. Franconi, M.C. Venier-Julienne, P. Menei, P. Jallet, J.J. Le Jeune, J.P. Benoit, Therapeutic efficacy of 5-fluorouracil-loaded microspheres on rat glioma: a magnetic resonance imaging study, NMR Biomed. 14 (6) (2001) $360-366$. 
26] K. Elkharraz, N. Faisant, C. Guse, F. Siepmann, B. Arica-Yegin, J.M. Oger, R. Gust, A Goepferich, J.P. Benoit, J. Siepmann, Paclitaxel-loaded microparticles and implants for the treatment of brain cancer: preparation and physicochemical characterization, Int. J. Pharm. 314 (2) (2006) 127-136.

[27] H. Zhang, S. Gao, Temozolomide/PLGA microparticles and antitumor activity against Glioma C6 cancer cells in vitro, Int. J. Pharm. 329 (1-2) (2007) 122-128.

[28] Y.H. Zhang, Z.J. Yue, H. Zhang, G.S. Tang, Y. Wang, J.M. Liu, Temozolomide/PLGA microparticles plus vatalanib inhibits tumor growth and angiogenesis in an orthotopic glioma model, Eur. J. Pharm. Biopharm. 76 (3) (2010) 371-375.

[29] M.E. Gil-Alegre, I. Gonzalez-Alvarez, L. Gutierrez-Pauls, A.I. Torres-Suarez, Three weeks release BCNU loaded hydrophilic-PLGA microspheres for interstitia chemotherapy: development and activity against human glioblastoma cells, J. Microencapsul. 25 (8) (2008) 561-568.

[30] W. Chen, D.R. Lu, Carboplatin-loaded PLGA microspheres for intracerebral injection: formulation and characterization, J. Microencapsul. 16 (5) (1999) 551-563.

[31] P. Menei, M. BoisdronCelle, A. Croue, G. Guy, J.P. Benoit, Effect of stereotactic implantation of biodegradable 5-fluorouracil-loaded microspheres in healthy and C6 glioma-bearing rats, Neurosurgery 39 (1) (1996) 117-123.

[32] P. Menei, L. Capelle, J. Guyotat, S. Fuentes, R. Assaker, B. Bataille, P. Francois, D. Dorwling-Carter, P. Paquis, L. Bauchet, F. Parker, J. Sabatier, N. Faisant, J.P. Benoit, Local and sustained delivery of 5-fluorouracil from biodegradable microspheres for the radiosensitization of malignant glioma: a randomized phase II trial, Neurosurgery 56 (2) (2005) 242-247.

[33] P. Menei, E. Jadaud, N. Faisant, M. Boisdron-Celle, S. Michalak, D. Fournier, M. Delhaye J.P. Benoit, Stereotaxic implantation of 5-fluorouracil-releasing microspheres in malignant glioma-a phase I study, Cancer 100 (2) (2004) 405-410.

[34] P. Menei, M.C. Venier, E. Gamelin, J.P. Saint-Andre, G. Hayek, E. Jadaud, D. Fournier, P. Mercier, G. Guy, J.P. Benoit, Local and sustained delivery of 5-fluorouracil from biodegradable microspheres for the radiosensitization of glioblastoma-a pilot study, Cancer 86 (2) (1999) 325-330.

[35] E. Fournier, C. Passirani, C. Montero-Menei, N. Colin, P. Breton, S. Sagodira, P. Menei, J.P. Benoit, Therapeutic effectiveness of novel 5-fluorouracil-loaded poly(methylidene malonate 2.1.2)-based microspheres on F98 glioma-bearing rats, Cancer 97 (11) (2003) 2822-2829

[36] V.G. Roullin, J.R. Deverre, L. Lemaire, F. Hindre, M.C. Venier-Julienne, R. Vienet, J.P. Benoit, Anti-cancer drug diffusion within living rat brain tissue: an experimenta study using [H-3](6)-5-fluorouracil-loaded PLGA microspheres, Eur. J. Pharm. Biopharm. 53 (3) (2002) 293-299.

[37] V.G. Roullin, M. Mege, L. Lemaire, J.P. Cueyssac, M.C. Venier-Julienne, P. Menei, E. Gamelin, J.P. Benoit, Influence of 5-fluorouracil-loaded microsphere formulation on efficient rat glioma radiosensitization, Pharm. Res. 21 (9) (2004) 1558-1563.

[38] M.S. Reza, T.L. Whateley, Iodo-2'-deoxyuridine (IUdR) and (125)IUdR loaded biodegradable microspheres for controlled delivery to the brain, J. Microencapsul. 15 (6) (1998) 789-801.

[39] G. Pradilla, P.P. Wang, P. Gabikian, K. Li, C.A. Magee, K.A. Walter, H. Brem, Loca intracerebral administration of paclitaxel with the Paclimer (R) delivery system: toxicity study in a canine model, J. Neuro-Oncol. 76 (2) (2006) 131-138.

[40] D.F. Emerich, S.R. Winn, R.T. Bartus, Injection of chemotherapeutic microspheres and glioma IV: eradicating tumors in rats, Cell Transplant. 11 (1) (2001) 47-54.

[41] D.F. Emerich, S.R. Winn, Y.H. Hu, J. Marsh, P. Snodgrass, D. LaFreniere, T. Wiens, B.P. Hasler, R.T. Bartus, Injectable chemotherapeutic microspheres and glioma I: enhanced survival following implantation into the cavity wall of debulked tumors, Pharm. Res. 17 (7) (2000) 767-775.

[42] D.F. Emerich, S.R. Winn, P. Snodgrass, D. LaFreniere, M. Agostino, T. Wiens, H. Xiong, R.T. Bartus, Injectable chemotherapeutic microspheres and glioma II: Enhanced survival following implantation into deep inoperable tumors, Pharm. Res. 17 (7) (2000) 776-781.

[43] E. Fournier, C. Passirani, N. Colin, P. Breton, S. Sagodira, J.P. Benoit, Development of novel 5-FU-loaded poly(methylidene malonate 2.1.2)-based microspheres for the treatment of brain cancers, Eur. J. Pharm. Biopharm. 57 (2) (2004) 189-197.

[44] M. Boisdroncelle, P. Menei, J.P. Benoit, Preparation and characterization of 5 fluorouracil-loaded microparticles as biodegradable anticancer drug carriers, J. Pharm. Pharmacol. 47 (2) (1995) 108-114

[45] O. Benny, M. Duvshani-Eshet, T. Cargioli, L. Bello, A. Bikfalvi, R.S. Carroll, M. Machluf, Continuous delivery of endogenous inhibitors from poly (lactic-co-glycolic acid) polymeric microspheres inhibits glioma tumor growth, Clin. Cancer Res. 11 (2) (2005) 768-776

[46] O. Benny, S.K. Kim, K. Gvili, I.S. Radzishevsky, A. Mor, L. Verduzco, L.G. Menon, P.M. Black, M. Machluf, R.S. Carroll, In vivo fate and therapeutic efficacy of PF-4/CTF microspheres in an orthotopic human glioblastoma model, FASEB J. 22 (2) (2008) 488-499.

[47] O. Benny, L.G. Menon, G. Arid, E. Goren, S.K. Kim, C. Stewman, P.M. Black, R.S. Carroll, M. Machluf, Local delivery of poly lactic-co-glycolic acid microspheres containing imatinib mesylate inhibits intracranial xenograft glioma growth, Clin. Cancer Res. 15 (4) (2009) 1222-1231

[48] T. Chandy, G.S. Das, G.H.R. Rao, 5-Fluorouracil-loaded chitosan coated polylactic acid microspheres as biodegradable drug carriers for cerebral tumours, J. Microencapsul. 17 (5) (2000) 625-638.

[49] T. Ozeki, K. Hashizawa, D. Kaneko, Y. Imai, H. Okada, Treatment of rat brain tumors using sustained-release of camptothecin from poly(lactic-co-glycolic acid) microspheres in a thermoreversible hydrogel, Chem. Pharm. Bull. (Tokyo) 58 (9) (2010) 1142-1147.

[50] T. Arai, O. Benny, T. Joki, L.G. Menon, M. Machluf, T. Abe, R.S. Carroll, P.M. Black, Novel local drug delivery system using thermoreversible gel in combination with polymeric microspheres or liposomes, Anticancer Res. 30 (4) (2010) 1057-1064.
[51] M. Hussain, G. Beale, M. Hughes, S. Akhtar, Co-delivery of an antisense oligonucleotide and 5-fluorouracil using sustained release poly (lactide-co-glycolide) microsphere formulations for potential combination therapy in cancer, Int. J. Pharm. 234 (1-2) (2002) 129-138.

[52] M. Yemisci, S. Bozdag, M. Cetin, F. Soylemezoglu, Y. Capan, T. Dalkara, I. Vural, Treatment of malignant gliomas with mitoxantrone-loaded poly (lactide-co-glycolide) microspheres, Neurosurgery 59 (6) (2006) 1296-1302 (discussion 1302-1293).

[53] D.F. Emerich, S.R. Winn, R.T. Bartus, Injection of chemotherapeutic microspheres and glioma III: Parameters to optimize efficacy, Cell Transplant. 11 (1) (2001) 35-45.

[54] T.T. Song, X.B. Yuan, A.P. Sun, H. Wang, C.S. Kang, Y. Ren, B. He, J. Sheng, P.Y. Pu, Preparation of injectable paclitaxel sustained release microspheres by spray drying for inhibition of glioma in vitro, J. Appl. Polym. Sci. 115 (3) (2009) 1534-1539.

[55] R. Lin, L. Shi Ng, C.H. Wang, In vitro study of anticancer drug doxorubicin in PLGAbased microparticles, Biomaterials 26 (21) (2005) 4476-4485.

[56] J.W. Xie, J.C.M. Marijnissen, C.H. Wang, Microparticles developed by electrohyd ro dynamic atomization for the local delivery of anticancer drug to treat C6 glioma in vitro, Biomaterials 27 (17) (2006) 3321-3332.

[57] J.W. Xie, R.S. Tan, C.H. Wang, Biodegradable microparticles and fiber fabrics for sustained delivery of cisplatin to treat C6 glioma in vitro, J. Biomed. Mater. Res. A 85A (4) (2008) 897-908.

[58] S.H. Ranganath, I. Kee, W.B. Krantz, P.K.H. Chow, C.H. Wang, Hydrogel matrix entrapping PLGA-paclitaxel microspheres: drug delivery with near zero-order release and implantability advantages for malignant brain tumour chemotherapy, Pharm. Res. 26 (9) (2009) 2101-2114.

[59] P.K. Naraharisetti, B.Y.S. Ong, J.W. Xie, T.K.Y. Lee, C.H. Wang, N.V. Sahinidis, In vivo performance of implantable biodegradable preparations delivering Paclitaxel and Etanidazole for the treatment of glioma, Biomaterials 28 (5) (2007) 886-894.

[60] K.W. Li, W.B. Dang, B.M. Tyler, G. Troiano, T. Tihan, H. Brem, K.A. Walter, Polilactofate microspheres for paclitaxel delivery to central nervous system malignancies, Clin. Cancer Res. 9 (9) (2003) 3441-3447.

[61] J.A. Floyd, A. Galperin, R. Ramakrishna, R. Rostomily, B.D. Ratner, Drug Encapsulated Aerosolized Microspheres as a Biodegradable, Intelligent Glioma Therapy, Society for Biomaterials 2014 Annual Meeting \& ExpositionDenver, CO, 2014.

[62] D.H.P. de la Ossa, M. Lorente, M.E. Gil-Alegre, S. Torres, E. Garcia-Taboada, M.D. Aberturas, J. Molpeceres, G. Velasco, A.I. Torres-Suarez, Local delivery of cannabinoid-loaded microparticles inhibits tumor growth in a murine xenograft model of glioblastoma multiforme, PLoS ONE 8 (1) (2013) e54795.

[63] T. Ozeki, D. Kaneko, K. Hashizawa, Y. Imai, T. Tagami, H. Okada, Improvement of survival in C6 rat glioma model by a sustained drug release from localized PLGA microspheres in a thermoreversible hydrogel (vol 427, pg 299, 2012), Int. J. Pharm. 430 (1-2) (2012) 393.

[64] D.Y. Zhang, A. Tian, X.X. Xue, M. Wang, B. Qiu, A.H. Wu, The effect of temozolomide/ poly(lactide-co-glycolide) (PLGA)/nano-hydroxyapatite microspheres on glioma U87 cells behavior, Int. J. Mol. Sci. 13 (1) (2012) 1109-1125.

[65] T. Araki, H. Yashima, K. Shimizu, T. Aomori, T. Hashita, K. Kaira, T. Nakamura, K. Yamamoto, Review of the treatment of non-small cell lung cancer with gefitinib, Clin. Med. Insights Oncol. 6 (2012) 407-421.

[66] J.A. Floyd, A. Galperin, B.D. Ratner, Drug Encapsulated Aerosolized Microspheres as a Localized, Degradable Cancer Therapy, 2014 AIChE Annual Meeting Atlanta, GA, 2014.

[67] E. Reguera-Nunez, C. Roca, E. Hardy, M. de la Fuente, N. Csaba, M. Garcia-Fuentes, Implantable controlled release devices for BMP-7 delivery and suppression of glioblastoma initiating cells, Biomaterials 35 (9) (2014) 2859-2867.

[68] L.D. Rhines, P. Sampath, F. DiMeco, H.C. Lawson, B.M. Tyler, J. Hanes, A. Olivi, H. Brem, Local immunotherapy with interleukin-2 delivered from biodegradable polymer microspheres combined with interstitial chemotherapy: a novel treatment for experimental malignant glioma, Neurosurgery 52 (4) (2003) 872-879 (discussion 879-880).

[69] S.J. Beer, C.B. Matthews, C.S. Stein, B.D. Ross, J.M. Hilfinger, B.L. Davidson, Poly(lacticglycolic) acid copolymer encapsulation of recombinant adenovirus reduces immunogenicity in vivo, Gene Ther. 5 (6) (1998) 740-746.

[70] P. Gabikian, B.M. Tyler, I. Zhang, K.W. Li, H. Brem, K.A. Walter, Radiosensitization of malignant gliomas following intracranial delivery of paclitaxel biodegradable polymer microspheres Laboratory investigation, J. Neurosurg. 120 (5) (2014) 1078-1085.

[71] T. Ozeki, D. Kaneko, K. Hashizawa, Y. Imai, T. Tagami, H. Okada, Combination therapy of surgical tumor resection with implantation of a hydrogel containing camptothecin-loaded poly(lactic-co-glycolic acid) microspheres in a C6 Rat glioma model, Biol. Pharm. Bull. 35 (4) (2012) 545-550.

[72] Y.H. Zhang, H. Zhang, J.M. Liu, Z.J. Yue, Temozolomide/PLGA microparticles: a new protocol for treatment of glioma in rats, Med. Oncol. 28 (3) (2011) 901-906.

[73] J. Dong, G.H. Zhou, D.F. Tang, Y.M. Chen, B.Q. Cui, X.L. Dai, J.S. Zhang, Q. Lan, Q. Huang, Local delivery of slow-releasing temozolomide microspheres inhibits intracranial xenograft glioma growth, J. Cancer Res. Clin. Oncol. 138 (12) (2012) 2079-2084.

[74] D. Allhenn, D. Neumann, A. Beduneau, Y. Pellequer, A. Lamprecht, A “drug cocktail" delivered by microspheres for the local treatment of rat glioblastoma, J. Microencapsul. 30 (7) (2013) 667-673.

[75] J. Hanes, A. Sills, Z. Zhao, K.W. Suh, B. Tyler, F. DiMeco, D.J. Brat, M.A. Choti, K.W. Leong, D.M. Pardoll, H. Brem, Controlled local delivery of interleukin-2 by biodegradable polymers protects animals from experimental brain tumors and liver tumors, Pharm. Res. 18 (7) (2001) 899-906.

[76] W. Hsu, M.S. Lesniak, B. Tyler, H. Brem, Local delivery of interleukin-2 and adriamycin is synergistic in the treatment of experimental malignant glioma, J. Neuro-Oncol. 74 (2) (2005) 135-140. 